\title{
VERBAL SHORT-TERM MEMORY REFLECTS THE ORGANIZATION OF LONG-TERM MEMORY. FURTHER EVIDENCE FROM SHORT-TERM MEMORY FOR EMOTIONAL WORDS.
}

\author{
Steve Majerus ${ }^{1,2} \&$ Arnaud D’Argembeau ${ }^{1,2}$ \\ ${ }^{1}$ Université de Liège, Belgium \\ ${ }^{2}$ Fonds de la Recherche Scientifique FNRS, Belgium \\ RUNNING HEAD: STM AND EMOTIONAL VALENCE \\ Journal of Memory and Language, 2011, 64, 181-197
}

\section{Address for correspondence:}

Steve Majerus, $\mathrm{PhD}$

Center for Cognitive and Behavioral Neuroscience

Boulevard du Rectorat, B33

4000 Liège, Belgium

Phone : 003243664656

Fax : 003243662808

Email : smajerus@ulg.ac.be 


\begin{abstract}
Many studies suggest that long-term lexical-semantic knowledge is an important determinant of verbal short-term memory (STM) performance. This study explored the impact of emotional valence on word immediate serial recall as a further lexico-semantic long-term memory (LTM) effect on STM. This effect is particularly interesting for the study of STM-LTM interactions since emotional words not only activate specific lexico-semantic LTM features but also capture attentional resources, and hence allow for the study of both LTM and attentional factors on STM tasks. In Experiments 1 and 2, we observed a robust effect of emotional valence on pure list recall in both young and elderly adults, with higher recall performance for emotional lists as opposed to neutral lists, as predicted by increased LTM support for emotional words. In Experiments 3 and 4 however, using mixed lists, it was the lists containing a minority of emotional words which led to higher recall performance over lists containing a majority of emotional words. This was predicted by a weak version of the attentional capture account. These data add new evidence to the theoretical position that LTM knowledge is a critical determinant of STM performance, with further, list-type dependent intervention of attentional factors.
\end{abstract}

197 words 
There is a substantial body of research showing that verbal short-term memory (STM) is not an autonomous cognitive function, but instead recruits linguistic knowledge bases to a very large extent. The present study explored a further potential long-term memory (LTM) effect on STM performance, the impact of emotional-semantic knowledge associated with emotional words.

\section{The impact of verbal LTM on STM}

Many studies show that the linguistic properties of the words, such as lexical status, lexical frequency, phonotactic frequency, word imageability, have a direct impact on their recall probability during a STM task (Gathercole, Frankish, Pickering \& Peaker, 1999; Hulme et al., 1997; Majerus \& Van der Linden, 2003; Roodenrys, Hulme, Alban, \& Ellis, 1994; Walker \& Hulme, 1999). The most prototypical effect is the lexicality effect, characterized by higher recall performance for lists composed of familiar words, as opposed to lists composed of nonwords (e.g., Gathercole et al., 1999; Hulme, Maughan \& Brown, 1991).These effects have been shown to be independent of articulatory rehearsal factors (e.g., Gathercole et al., 1999; Hulme et al., 1991). The existence of these psycholinguistic effects has been interpreted as reflecting the influence of phonological, lexical and semantic language representations stored in LTM on verbal STM (Hulme et al., 1991). On the one hand, this LTM support is supposed to function at the level of retrieval, when the decayed STM traced is reconstructed via corresponding LTM representations: the more available and familiar these representations are, the more efficient these reconstruction processes will be (the so-called redintegration process) (Hulme et al., 1997; Gathercole et al., 1999; Schweickert, 1993). Other theoretical accounts consider that the impact of language knowledge is operational during all STM stages, temporary STM traces being immediately supported by feedback connections between the language system and its knowledge 
bases and the verbal STM system (e.g., Burgess \& Hitch, 1999; Gupta, 2003; N. Martin \& Saffran, 1992; R. Martin, Shelton, \& Yafee, 1994).

The impact of semantic knowledge, most closely related to the emotional-semantic LTM features under investigation in this study, has been studied mainly via the exploration of word concreteness and semantic relatedness effects on STM performance. Walker and Hulme (1999) assessed word concreteness effects, by comparing ISR for high imageability versus low imageability words, observing a small but significant advantage for recall of high imageability words, suggesting that the richer semantic content defining high imageable words provides stronger semantic support during word recall in STM tasks (Walker \& Hulme, 1999; see also Majerus \& Van der Linden, 2003). Poirier and Saint-Aubin (Poirier \& Saint-Aubin, 1995; SaintAubin \& Poirier, 1999) showed that word lists composed of semantically related words yield higher recall performance than words lists containing semantically unrelated words, the so-called semantic relatedness effect. Importantly, the studies by Poirier and Saint-Aubin show that semantic LTM support does not benefit all aspects of STM recall. By distinguishing item and order recall performance, the authors observed that recall of item information (i.e., the words to be recalled, irrespectively of their serial position) is most strongly influenced by semantic knowledge, while recall of order information (i.e., the serial position of the words in the list) is relatively insensitive to semantic (as well as to most other psycholinguistic) variables (see also Nairne \& Kelley, 2004). At a more general theoretical level, these results are consistent with recent models considering that the verbal long-term knowledge stored in the language system is providing the representational substrate for storing item information in a STM task, while order information is processed and stored via a dedicated STM system, distinct from the language system (e.g., Burgess \& Hitch, 1999; Gupta, 2003; Majerus, Heiligenstein, Gautherot, Poncelet \& Van der Linden, 2009; Martin et al., 1994). It follows that recall of item information, being 
directly linked to the processing properties of the language system, should be sensitive to all types of long-term verbal knowledge, while order information will be less sensitive to these verbal LTM effects.

\section{The impact of emotional semantic content on STM}

The aim of the present study was to explore the impact of emotional versus nonemotional semantic word status as a further potential LTM effect in STM. Emotional words, such as words having a positive or negative valence like 'pleasure, love, winner' versus 'loser, discomfort, sickness', form a special semantic category in the sense that the underlying semantic representation can be considered to be richer relative to neutral words, because of the additional emotional and motivational polarity which will induce an automatic categorization of the words according to a positivity / negativity dimension (e.g., Bradley, 2000). Many studies outside the STM domain have shown that emotional words elicit selective responding relative to neutral words, such as in lexical decision, naming, automatic response inhibition and vigilance tasks (Algom, Chajut, \& Lev, 2004; Estes \& Verges, 2008; Kanske \& Kotz, 2007; Kousta, Vinson, \& Vigliocco, 2009; Larser, Mercer \& Balota, 2006; Pratto \& John, 1991). This effect of emotional words cannot be simply reduced to an enhanced word concreteness effect. Although both emotionality and concreteness have been shown to modulate event-related brain responses involved in semantic processing (the N400 component), emotionality and concreteness modulate this response independently (Kanske \& Kotz, 2007). In other words, both concreteness and emotionality lead to enhanced semantic processing, but emotionality does not simply arise from enhanced concreteness. Furthermore, lists of positive words or lists of negative words are also likely to be semantically related to a stronger degree than randomly selected neutral words since they have in common a positive or negative semantic dimension. For example, negative words 
have all in common the fact that they refer to events and states characterized by adversity (e.g., danger, disease, pain, disgust, accident, sadness). Positive words on the other hand are associated with semantic concepts that can be broadly qualified as hedonistic and life enhancing (e.g., marriage, joy, money, holidays, meal). Hence, in addition to showing richer semantic content, emotional words are also likely to show stronger semantic relatedness, relative to random neutral words matched for frequency and concreteness. In the LTM domain, studies have shown that the effects of emotional words arise, at least in part, from semantic relatedness (Talmi \& Moscovitch, 2004). With respect to STM tasks, since emotionality is associated with richer semantic processing and greater semantic relatedness, we should expect that the higher the number of emotional words in a STM list, the higher recall performance, resulting in the largest effect of emotion when comparing recall for pure lists composed of negative or positive words relative to lists of neutral words.

However, stronger semantic support is not the only dimension that underlies the effect of emotion in cognitive tasks. Emotion processing is itself a specific psychological function involved in the regulation of behavior and, ultimately, survival (Damasio, 2001). Although emotional words do not necessarily activate intense negative or positive feelings when being presented, they might nevertheless engage to some extent the motivational systems involved in emotion processing: the approach-appetitive system, for positive words, and the withdrawalaversive system, for negative words (Bradley, 2000). Although there is currently a controversy going on with respect to the possible dominance of the withdrawal-aversive system over the approach-appetitive system (Kousta et al., 2009; Lang, Bradley \& Cuthbert, 1997; Pratto \& John, 1991), most studies controlling for possible confounding psycholinguistic factors such as word frequency and orthographic neighborhood observe that both positive and negative stimuli lead to preferential processing over neutral stimuli, as predicted by the model of motivated attention and 
affective states (Lang et al., 1997). In these studies, facilitated processing for emotional words is attributed to attentional factors: emotional words are processed faster because they attract and hold attention to a greater extent than neutral words.

This makes the study of the impact of emotional valence on verbal STM recall particularly interesting: if emotional words not only have a specific LTM semantic content facilitating their processing relative to neutral words, but also preferentially attract attention, how do these LTM and attentional factors interact during a verbal STM task? In a pure list design, the attention 'grabbing' effect for emotional words should have a relatively negligible influence since all words of the memory list will attract attention and since the number of words than can be simultaneously held in the focus of attention is limited (e.g., Cowan, 1995). The effect of attentional 'grabbing' should thus be maximal in a mixed list context, where a minority of emotional words is presented within a list containing a majority of neutral words. In this situation, the emotional words will preferentially capture attention, and facilitate encoding and recall (Christianson \& Engelberg, 1999; Pesta, Murphy, \& Sanders, 2001; see also McKay et al., 2004, for a similar account involving preferential episodic context binding mechanisms for emotional words rather than attentional orienting processes).

Currently, there is very limited evidence for or against an impact of emotional content on STM performance, in either pure or mixed list contexts. A study by Kensinger and Corkin (2003) investigated the impact of negative words on various working memory tasks (e.g., N-back task, backward recall) using lists composed of exclusively negative or exclusively neutral words. No effect of emotional valence was observed in that study using pure list designs. However, the high working memory demands used in these tasks and the involvement of executive and strategic processes necessary for updating and stimulus manipulation render the interpretation of this null result somewhat difficult. Monnier and Syssau (2008), using a pure list design and simple ISR 
tasks, observed indeed better recall performance for pleasant (positive) word lists as compared to neutral word lists. Possible differences in semantic relatedness between the two list types were not controlled, however, so the specific mechanisms underlying the detected difference in STM recall remain unclear. Furthermore, only positive word lists were used in that study, leaving unknown whether the observed effect would generalize to words with negative content. Tse and Altarriba (2009) observed higher recall performance for negative word lists relative to neutral word lists, while observing no advantage for positive word lists relative to neutral word lists. Ferré (2002) observed an effect of emotional valence on immediate serial performance within a mixed list design, positive and negative words being recalled more accurately than neutral words.

In sum, few studies have directly investigated the impact of emotional content on verbal STM recall performance, and the limited and partially conflicting evidence that is available does not allow us to determine whether this effect is due to the LTM semantic properties of emotional words and to what extent the attentional 'grabbing' properties of emotional words further affect STM performance for emotional words. We explored these questions in four experiments. In Experiments 1 and 2, we explored immediate serial recall for pure lists of positive, negative and neutral words in order to determine whether there is an effect of emotional content on pure word list recall, as predicted by stronger semantic LTM support for emotional words. Experiments 3 and 4 explored recall performance for mixed lists, containing either a minority of emotional words, or a minority of neutral words. If the effect of emotion on STM is related to the attentional properties of emotional words rather than to their semantic LTM characteristics per $s e$, then no effect or only a very mild effect of emotional valence should be observed in Experiments 1 and 2, but a strong effect should be observed in Experiments 3 and 4 for lists containing a minority of emotional words. 


\section{EXPERIMENT 1: ISR FOR PURE LISTS OF EMOTIONAL WORDS IN YOUNG ADULTS}

The first experiment assessed the impact of emotional content on pure list recall using a standard ISR procedure for 6-item word lists by explicitly controlling for semantic relatedness. In each list, all words either had a negative valence, a positive valence or a neutral valence. Furthermore, we applied the item-order distinction for assessing the impact of emotionality on word list recall. As noted in the Introduction, the impact of linguistic knowledge on verbal STM is strongest for item recall, as opposed to order recall, since coding of item in a STM task is supposed to recruit to a large extent the language system; order recall on the other hand is supposed to depend on a specialized and functionally distinct STM system, independent from verbal long-term knowledge (e.g., Burgess \& Hitch, 1999; Gupta, 2003; Majerus et al., 2006; 2009; Majerus, 2009). Hence we expected that the effect of emotional semantic content on STM recall is greatest for item recall as opposed to order recall. Within-list semantic relatedness was controlled by obtaining semantic relatedness ratings for all adjacent word pairs occurring in the different lists (see also Tse and Altarriba, 2009). Like in the only study that observed a reliable effect of emotional content on ISR performance for pure lists, stimuli were presented auditorily (Monnier \& Syssau, 2008). However, contrary to Monnier and Syssau, all stimuli were prerecorded sound files in order to ensure standardized presentation format.

Methods

Participants 
16 young adults ( 8 female) aged between 24 and 30 years participated in this experiment. All participants were native French speakers and presented no history of developmental, learning or medical difficulties. Informed written consent was obtained for all participants.

Material

In a pilot study, we built up a database of 690 words by selecting words from a database containing the affective value of French words (Messina, Morais, \& Cantraine, 1989) and by translating words from an English database (Bradley \& Lang, 1999) into French. The selected words were then rated by twenty-four healthy young adults (aged between 18 and 32 years) for imageability, emotional intensity, and emotional valence. The two emotional dimensions were rated using the 9-point Self Assessment Manikin Scales (Bradley \& Lang, 1994) and imageability was rated with a 9-point Likert scale adapted from Paivio, Yuille, and Madigan (1968). Based on these ratings, 90 neutral words, 90 words with a positive emotional valence and 90 words with a negative emotional valence were selected from the same database of 690 words. The words in the three conditions significantly differed for emotional valence, $F(2,267)=5358$, $\mathrm{p}<.0001$, and emotional intensity, $\mathrm{F}(2,267)=34.42, \mathrm{p}<.0001$, while being matched for lexical frequency, $\mathrm{F}(2,267)<1$, n.s., for word imageability, $\mathrm{F}(2,267)<1$, n.s., and for number of syllables, $\mathrm{F}(2,267)<1$, n.s. (Lexique 2 - frantext ratings; New et al., 2004) (see Table 1 for mean values and ranges). The words were recorded by a female voice on digital disc using a monotonous voice, and edited into single .wav files for analysis and storage on a PC compatible computer. The stimuli were matched for spoken duration across the three stimulus conditions, $F(2,267)<1$, n.s. (see Table 1 for values). For each condition, 15 lists containing 6 words each were constructed by sampling without replacement from the respective pool of items and by avoiding item pairs that showed obvious semantic relatedness (e.g., house - window). The recordings of 
the selected words were concatenated to create 6-item lists, at the rate of one word every 2 seconds [e.g., positive word list: espoir (hope) - progrès (progress) - concert (concert) - trophée (trophy) - loisir (leisure) - charme (charm); negative word list: bruit (noise) - ridicule (ridiculousness) - collision (collision) - rejet (rejection) - cadavre (corpse) - trafic (traffic); neutral word lists: secrétaire (secretary) - contexte (context) - odeur (odor) - transfert (transfer) - habitant (inhabitant) - anse (handle)] . This procedure was repeated once, generating for each condition two sets of 15 word lists. Half of the participants were administered the first set, and half of the participants were administered the second set. For each set, within-list semantic associations were controlled by administering the lists to 15 healthy young adults, not participating in the ISR experiments; they rated adjacent item pairs of all lists for semantic relatedness, on a scale ranging from 0 (no relation) to 5 (very highly related or synonym). Mean modes of semantic relatedness ratings were very low and equal or close to 0 in all conditions (means and standard deviations: neutral lists : $0 \pm 0.00$ for set $1,0 \pm 0.00$ for set 2 ; positive lists: $0.41 \pm 0.60$ for set $1,0.45 \pm 0.57$ for set 2 ; negative lists: $0.53 \pm 0.74$ for set $1,0.31 \pm 0.43$ for set 2 ). Nevertheless, as can be seen from semantic relatedness ratings, although remaining very close to 0, semantic relatedness modes were nevertheless somewhat higher for negative and positive lists, as opposed to neutral lists were the mode equaled invariably to 0 . As already developed in the Introduction section, this was expected given that emotional words will always have minimal semantic relatedness relative to neutral words since they are sharing positive or negative emotional category membership.

\section{< INSERT TABLE 1 ABOUT HERE >}

Procedure 
The lists were presented via headphones connected to a portable PC. The participants were instructed that they would hear 6 words presented via the headphones, and they were asked to recall the words in correct order immediately after presentation. After the participant's response, the examiner activated presentation of the next list. Each participant's responses were digitally recorded and stored on computer disk for later transcription and scoring. The conditions were presented in blocks, the order of blocks being randomized for each participant. For each condition, we determined the number of words recalled in correct serial position, the number of item errors (omissions, phonological, semantic or unrelated intrusions) and the number of order errors (words recalled in the wrong serial position) by pooling over the six serial positions.

\section{Results and discussion}

A first repeated measures ANOVA assessed the impact of emotional valence on the number of words correctly recalled over the six serial positions. No main effect of emotional condition was observed, $\mathrm{F}(2,30)=1.32, \mathrm{MSE}=0.02, \mathrm{p}=.28, \eta_{\mathrm{p}}^{2}=0.08$ (see Table 2 for means and standard deviations of recall performance as a function of list type). A second ANOVA determined the impact of emotional valence on item errors. A significant effect of emotional valence was observed, $F(2,30)=5.24, \mathrm{MSE}=7.46, \mathrm{p}<.01, \eta^{2}{ }_{\mathrm{p}}=0.26$ (see Figure 1). Post-hoc comparisons (Bonferroni corrections) showed a significantly higher amount of item errors for neutral word lists as compared to positive word lists and negative word lists. A final ANOVA revealed a non-significant impact of emotional valence on order errors, $F(2,30)=2.74$, $\mathrm{MSE}=7.09, \mathrm{p}=.08, \eta_{\mathrm{p}}^{2}=0.15$. Post-hoc comparisons revealed no significant differences between the neutral condition and the two emotional conditions. 


\section{< INSERT TABLE 2 AND FIGURE 1 ABOUT HERE >}

An impact of emotional valence was observed for pure list recall, and this only for item errors, recall of positive and negative word lists leading to better item recall than recall of neutral word lists. This finding is consistent with the prediction that item recall but not order recall should benefit from the richer semantic characteristics associated with emotional words. Most importantly, these results obtained by using a pure list design are in line with the prediction of enhanced semantic LTM support for emotional words during STM tasks.

\section{EXPERIMENT 2: ISR FOR PURE LISTS OF EMOTIONAL WORDS IN YOUNG AND ELDERLY ADULTS}

The aim of Experiment 2 was to replicate the results of Experiment 1, by controlling for a potentially confounding variable in Experiment 1: intonational contour. Although the experimenter recording the words had been instructed to use a monotonous voice, intonational contours might have been unintentionally modulated for some of the words (e.g., higher amplitude and lengthening for all or some syllables of a word); these potential intonational specificities of some of the words could have influenced STM performance by selectively attracting attention, and diverting attention from other items in the list. This possibility was controlled by determining the pitch contour, amplitude and pronunciation times for each recording of the words used in Experiment 2. Furthermore, in order to increase the likelihood of obtaining strong effects of emotional content on ISR performance especially for word lists with a positive valence, we included a group of elderly participants. Elderly adults, as compared to 
young adults, have been shown to present a stronger sensitivity towards stimuli with a positive valence in a range of LTM and attentional tasks (for review, see Mather \& Carstensen, 2005). Extending these observations to the STM domain, a robust impact of positive valence on ISR performance should be most likely in elderly adults. Finally, as in Experiment 1, semantic relatedness of all stimuli was controlled by obtaining semantic relatedness judgments for all stimulus pairs of each list.

\section{METHODS}

Participants

Fifteen young adults ( 8 female) aged between 24 and 30 years, and 15 elderly adults ( 8 female) aged between 60 and 84 years participated in this experiment. All participants were native French speakers and presented no history of developmental, learning or current major medical difficulties.

The Positive and Negative Affective Schedule (PANAS; Watson, Clark, \& Telegen, 1988; French adaptation by Gaudreau, 2000) was used to assess positive and negative affect, since young and elderly participants might differ in affective states. The PANAS contains adjectives describing ten positive and ten negative affects, for which participants are instructed to rate their current state using a five-point Likert-type scale from 1 (not at all or a little) to 5 (extremely). The young participants showed overall higher scores on the PANAS dimensions, $\mathrm{F}(1,28)=4.17, \mathrm{MSE}=35.34, \mathrm{p}=.05, \eta_{\mathrm{p}}^{2}=.13$, all participants scored higher on the positive than the negative dimensions, $F(1,28)=117.97, \mathrm{MSE}=22.60, \mathrm{p}<.0001, \eta^{2}{ }_{\mathrm{p}}=.18$, and the young participants showed higher scores than the elderly participants most specifically for the positive 
dimension (mean: $30.27 \pm 5.23$ and $24.53 \pm 5.64$, for young and elderly groups, respectively), while scores were comparable for the negative dimension in both groups, (mean: $14.33 \pm 5.27$ and $13.80 \pm 5.37$, for young and elderly groups, respectively), $\mathrm{F}(1,28)=4.49, \mathrm{MSE}=22.60, \mathrm{p}<.05, \eta^{2} \mathrm{p}$ $=.14)$. Informed written consent was obtained for all participants.

\section{Material}

One-hundred twenty neutral words, 120 words with a positive emotional valence and 120 words with a negative emotional valence were selected from a database of 690 words rated for word imageability, emotional intensity and emotional valence (see Experiment 1). The words in the three conditions significantly differed for emotional valence, $F(2,357)=11703$, $p<.0001$, and emotional intensity, $\mathrm{F}(2,357)=77.45$, $\mathrm{p}<.0001$, while being matched for lexical frequency, $\mathrm{F}(2,357)<1.96$, n.s. and for word imageability, $\mathrm{F}(2,357)=1.71$, n.s. (see Table 3). Since the database from which the words were selected is derived from judgments of emotional valence given by young adults, we also checked whether elderly participants rate the words in a similar way. We administered all the words used in this experiment to the group of elderly participants, after they had been administered the immediate serial recall tasks. They had to rate all the words for emotional valence, using the same procedure as described in Experiment 1. As shown in Table 3, nearly identical mean values for emotional valence were observed in elderly participants and young adults: the words in the three conditions also significantly differed for emotional valence, $\mathrm{F}(2,357)=3339.50, \mathrm{p}<.0001$, when considering the ratings obtained by the elderly participants. Finally, there was a small but significant effect for the number of syllables, $\mathrm{F}(2,357)=4.64, \mathrm{p}<.05$. This small difference in the theoretical number of syllables however had 
no effect on actual pronunciation times, as shown below. Lexical frequency estimates and syllable counts were obtained from the database Lexique 2 - frantext ratings (New et al., 2004).

The words were recorded by a female voice on digital disc, and edited into single .wav files for analysis and storage on a PC compatible computer. For each word and each syllable, minimum and maximum pitch were determined using Praat speech analysis software (Boersma \& Weenink, 2001); pronunciation duration and mean amplitude for each entire word were further determined. As shown in Table 3, minimum and maximum pitch was matched for first, second, and third syllables of the words of each of the three conditions; mean word duration was also perfectly matched. All words had a mean amplitude of $71 \mathrm{~dB}$. For each condition, 20 lists containing 6 words each were constructed by sampling without replacement from the respective pool of items and by avoiding item pairs that showed obvious semantic relatedness. The recordings of the selected words were then concatenated to create 6-item lists, at the rate of one word every 2 seconds. [e.g., positive word list: triomphe (triumph) - chance (luck) - lac (lake) trésor (treasure) - encouragement (encouragement) - harmonie (harmony); negative word list: noyade (drowning) - poignard (dagger) - horreur (horror) - migraine (migraine) - ennui (boredom) - avalanche (avalanche); neutral word lists: pièce (piece) - habitude (habit) tendance (tendency) - pion (pawn) - estimation (estimation) - bocal (jar)] . For each set, withinlist semantic associations were formally checked by administering the lists to 15 healthy young adults, not participating in the ISR experiments; they rated adjacent item pairs of all lists for semantic relatedness, on a scale ranging from 0 (no relation) to 5 (very highly related or synonym). Mean modes of semantic relatedness ratings were very low and equal or close to 0 in all conditions (neutral lists: $0 \pm 0.00$; positive lists: $0.30 \pm 0.58$; negative lists: $0.75 \pm 1.05$ ). As in Experiment 2, semantic relatedness modes were nevertheless somewhat higher for negative and positive lists, as opposed to neutral list where the mode invariably equaled to 0. 


\section{$<$ INSERT TABLE 3 ABOUT HERE >}

Procedure

The lists were presented via headphones connected to a portable PC. The participants were instructed that they would hear 6 words presented via the headphones, and they were asked to recall the words in correct order immediately after presentation. After the participant's response, the examiner activated presentation of the next list. Each participant's responses were digitally recorded and stored on computer disk for later transcription and scoring. The different list conditions were presented in blocks, the order of blocks being randomized for each participant. For each condition, we determined the number of words recalled in correct serial position, the number of item errors (omissions, intrusions, phonological or semantic paraphasias) and the number of order errors (words recalled in the wrong serial position) by pooling over the six serial positions.

Results and discussion

A first $2 * 3$ mixed ANOVA assessed the impact of emotional valence (within-subjects factor) on the number of words recalled in correct serial position, as a function of age group (between-subjects factor). We observed an effect of emotional condition, $\mathrm{F}(2,56)=19.01$, $\mathrm{MSE}=0.01, \mathrm{p}<.001, \eta_{\mathrm{p}}^{2}=0.40$ and an effect of age group, $\mathrm{F}(1,28)=18.28, \mathrm{MSE}=0.14, \mathrm{p}<.001, \eta^{2}$ $\mathrm{p}=0.39$ (see Table 4 for mean values and standard deviations). Furthermore, there was a marginally significant group by emotional valence interaction, $\mathrm{F}(2,56)=3.12, \mathrm{MSE}=0.01, \mathrm{p}=.05$, $\eta_{p}^{2}=0.10$. Post-hoc comparisons (with Bonferroni corrections) showed overall higher recall for 
positive words relative to both neutral word and negative word condition (all p's <.0001); recall for negative and neutral words did not significantly differ. Furthermore, as can be seen in Table 4, the difference between positive and neutral conditions was slightly increased in the group of elderly adults.

\section{$<$ INSERT TABLE 4 ABOUT HERE >}

A second $2 * 3$ mixed ANOVA assessed the same effects but specifically for item errors. As in the preceding analysis, we observed a main effect of emotional condition, $\mathrm{F}(2,56)=17.14$, $\mathrm{MSE}=28.5, \mathrm{p}<.001, \eta_{\mathrm{p}}^{2}=0.38$ and an effect of age group, $\mathrm{F}(1,28)=15.92, \mathrm{MSE}=391.40, \mathrm{p}<.001$, $\eta_{p}^{2}=0.36$. The group by emotional valence interaction was not significant, $F(2,56)=1.84$, $\mathrm{MSE}=28.50, \mathrm{p}=.17, \eta_{\mathrm{p}}^{2}=0.06$. Post-hoc comparisons (with Bonferroni corrections) showed again an overall lower amount of item errors for positive words relative to both neutral word and negative word condition, (all p's $<.001$ ); item errors for negative and neutral words did not differ (see Figure 2). A final $2 * 3$ mixed ANOVA assessed the impact of emotional content on order errors. A marginally significant effect of age group was observed, $F(1,28)=4.04$, $\mathrm{MSE}=584.40, \mathrm{p}=.05, \eta_{\mathrm{p}}^{2}=0.13$. The effect of emotional content, $\mathrm{F}(2,56)<1, \mathrm{MSE}=21.36, \mathrm{p}=.44$, $\eta_{p}^{2}=0.03$ and the interaction, $F(2,56)=1.36, M S E=28.5, p=.26, \eta_{p}^{2}=0.05$ were non-significant.

\section{$<$ INSERT FIGURE 2 ABOUT HERE >}

In sum, Experiment 2 revealed a robust and consistent effect of emotional content on ISR performance, both groups showing significant effects of emotional valence on ISR performance. Also, as in Experiment 1, this effect was significant only for recall of item information, but not order information. This is in line with other verbal LTM effects on STM performance, the impact 
of language knowledge being most consistent for item recall as opposed to order recall. Unlike Experiment 1, the effect of emotional valence was significant only for positive word lists, while in the first Experiment, better item recall was observed for both positive and negative word lists, as opposed to neutral word lists. The recordings of stimuli in Experiment 2 being more rigorously controlled for intonation patterns, one could argue that the effect observed for negative words in Experiment 1 was related to potential uncontrolled differences in intonation patterns for negative words. However, a posteriori analysis of intonation patterns revealed no difference between recordings for neutral and negative stimuli in Experiment 1 (neutral stimulisyllable 1: mean pitch $_{\min }=216 \mathrm{~Hz}$, mean pitch $_{\max }=259 \mathrm{~Hz}$; negative stimuli - syllable $1:$ mean pitch $_{\min }=214 \mathrm{~Hz}$, mean pitch $_{\max }=257 \mathrm{~Hz}$; neutral stimuli - syllable $2:$ mean pitch $_{\min }=207 \mathrm{~Hz}$, mean pitch $_{\max }=250 \mathrm{~Hz}$; negative stimuli - syllable 2: mean pitch $_{\min }=209 \mathrm{~Hz}$, mean pitch $_{\max }=250 \mathrm{~Hz}$ ).

Finally, we found that the impact of positive emotional valence on the number of words recalled in correct serial position was somewhat stronger in elderly participants as compared to young participants, which is in line with the hypothesis that elderly people present a positivity bias (Mather \& Carstensen, 2005). On the other hand, contrary to this hypothesis, there was no age by emotional valence interaction for item errors. Some previous studies exploring the positivity bias in LTM experiments also did not observe a differential positivity effect in elderly participants relative to young participants (Comblain, D’Argembeau, Van der Linden, \& Aldenhoff, 2004; Denburg, Buchanan, Tranel, \& Adolphs, 2003; Kensinger et al., 2002). It should be noted that the (lack of) age-related differences that was observed in this study is difficult to interpret because the two groups differed in terms of positive affect (as assessed by PANAS questionnaire), the young group rating higher on the positive dimension than the elderly group . However, the important point for our purpose is that there was a reliable effect of positive emotional content on ISR performance in both age groups. 


\section{EXPERIMENT 3: ISR FOR MIXED LISTS OF EMOTIONAL WORDS IN YOUNG AND ELDERLY PARTICIPANTS}

Experiments 1 and 2 show reliable effects of emotional content on pure list recall, while controlling for a number of possible confounding variables (lexical frequency, word imageability, word length, stimulus duration, intonation patterns). We also controlled for semantic relatedness between stimulus pairs, by minimizing semantic relatedness in all three list conditions (positive, negative, neutral). However, as already acknowledged, although semantic relatedness was minimized, there was yet some minimal residual semantic relatedness within lists of positive words and within lists of negative words. Although it is unlikely that this residual semantic relatedness explains the observed patterns of results (in Experiment 2, an effect of emotional content was observed for positive but not negative word lists, while both lists had the same degree of minimal semantic relatedness), we controlled for this possibility by running a third experiment using mixed list designs. If the effect of emotional content we have observed in pure lists is only due to residual semantic relatedness, then this effect should disappear when mixing emotional and neutral words and completely removing any residual between stimuluspair semantic relatedness.

The second aim of this Experiment was to explore the impact of attentional "grabbing" processes on processing emotional stimuli during a STM task. As described in the Introduction, the emotional-semantic content associated with emotional words is characterized by a privileged connection with attentional processes: emotional words capture attentional processes to a greater extent than neutral stimuli when emotional and neutral words are presented together in the same context (Lang et al., 1997; Huang, Baddeley, \& Young, 2008). This should be especially the case in mixed lists, where emotional and neutral words are alternated, allowing preferential 
attentional orientation towards emotional words, especially when there are only a few emotional words. Thus, in mixed lists, when there is also an intervention of attentional factors, we should expect a reversed 'emotionality' effect: emotional words in lists containing a majority of emotional words should actually lead to lower recall performance as opposed to emotional words in lists containing a minority of emotional words. This should be so because in mixed lists containing a minority of emotional words, the emotional words will benefit from the intervention of both LTM and preferential attentional orientation, while only LTM factors will intervene in the case of mixed lists containing a majority of emotional words. The emotional words in emotion-infrequent lists will preferentially attract attention, while in a list containing a majority of emotional words, attention will be attracted by all emotional words and hence the effect of preferential attentional orientation will be diluted as the limited attentional resources have to be divided among the many emotional words that all 'grab' attention simultaneously.

However, two possible outcomes for recall performance in emotion-infrequent lists have to be distinguished here. The strongest prediction would be to assume that emotional words will capture all available attentional resources, leading to a strong advantage of emotion words over neutral words in emotion-infrequent lists. We will call this account the strong attentional account. This is in accordance with the attentional account proposed for emotional words mainly based on evidence from rapid visual presentation paradigms (Barnard, Ramponi, Battye, \& Mackintosh, 2005; Huang et al., 2008). In these paradigms, an attentional blink effect is observed for neutral stimuli following an emotional stimulus: neutral stimuli will be less likely to be recalled in that case. However, it has also been shown that this attentional blink effect is very short (between 100-300 msec) (e.g. Barnard et al., 2005; Huang et al., 2008). Hence, at longer intervals typically used in verbal STM experiments, it is less likely that attention will still be completely captured by the preceding emotional stimulus when a neutral stimulus occurs (see 
also Hadley \& MacKay, 2004, for a similar account). Hence, a second possibility is that the preferential attentional capture will not consume all attentional resources, but the heightened attentional focus on emotional words instead will have a more general effect on list recall for both emotional and neutral words by providing additional cues for parsing the STM list. Items with heightened attentional focus will stand out from the other items in the list, facilitating recall for these emotional items (e.g., Jones \& Yee, 1993). At the same time, these items, being marked by enhanced attentional capture, will allow for more efficient parsing of the STM list as they can be used to segment the STM list in different parts. Neutral items can then be grouped around these attentional markers of the STM list, leading to facilitated recall performance for both emotional and neutral items. We will call this the weak attentional account, predicting enhanced recall performance for both neutral and emotional words in emotion-infrequent lists. Partial support for this hypothesis comes from studies where a minority of positions and/or items have been marked, either at the perceptual or the phonological level (e.g., a minority of positions with enhanced word stress; one long word among many short words). In these studies, recall not only increased for enhanced stimuli/positions but also for the other stimuli/positions (Hulme, Stuart, Surprenant, Neath, Shostak, \& Brown, 2006; Reeves, Schmauder \& Morris, 2000). While Hulme et al. interpreted these results in the context of distinctiveness, Reeves et al. considered that the marked positions allowed for grouping of marked and adjacent unmarked positions (see also Frankish (1995)). However, it should be noted that the studies by Reeves et al. and Frankish focused on positional marking rather than on item marking per se. Finally, if emotional items lead to more efficient parsing of the STM list, allowing for binding of the neutral words with the adjacent emotional word, then the emotional items can also act as efficient retrieval cues for the neutral words at recall. In sum, the weak attentional account considers that the emotional words in emotion-infrequent lists act as attentional markers of the STM list, providing efficient cues for 
parsing of the list during encoding, and for item retrieval during recall, leading to a benefit for recall performance of both emotional and neutral items.

With respect to item and order errors, the strong attentional account will predict enhanced item recall since emotional words in emotion-infrequent lists, will maximally grab attention. For order errors on the other hand, a trade-off between item and order encoding processes may occur. Attention being fully captured by the emotional content of emotional items, encoding of emotional items may be favored, to the detriment of other items and list-level serial position information (order-encoding hypothesis: Delosh \& MacDaniel, 1996; see also Mulligan, 2002). According to the weak attentional account, enhanced item recall will also be predicted for emotion-infrequent lists, but for both emotional and neutral stimuli. Furthermore, no disruption is expected for order recall since attention is not diverted from the neutral items and the general list-level information including serial position information. Given the additional markers in emotion-infrequent lists which facilitate segmentation of the STM list, as assumed by the weak attentional account, one may perhaps even expect a decrease of order errors in this condition.

\section{METHODS}

\section{Participants}

Fifteen young adults ( 8 female) aged between 24 and 30 years, and 15 elderly adults ( 8 female) aged between 60 and 84 years participated in this experiment. All participants were native French speakers and presented no history of developmental, learning or current major medical difficulties.

As in Experiment 2, the Positive and Negative Affective Schedule (PANAS; Watson, Clark, \& Telegen, 1988; French adaptation by Gaudreau, 2000) was used to assess any potential 
group differences in positive and negative affect. Young and elderly participants showed similar scores on the PANAS dimensions overall, $F(1,26)<1.00, \mathrm{MSE}=41.12$, n.s., $\eta_{p}^{2}=.01$, all participants scoring higher on the positive than the negative dimensions, $\mathrm{F}(1,26)=111.25$, MSE $=28.15, \mathrm{p}<.0001, \eta_{\mathrm{p}}^{2}=.81$, and the young participants showing comparable scores to the elderly participants for the positive dimension (mean: $27.00 \pm 7.11$ and $26.87 \pm 7.61$, for young and elderly groups, respectively), and the negative dimension, (mean: $11.08 \pm 1.66$ and 12.80 \pm 5.00 , for young and elderly groups, respectively), $\mathrm{F}(1,26)<1.00, \mathrm{MSE}=28.15$, n.s. $\left.\eta_{\mathrm{p}}^{2}=.02\right)$. Informed written consent was obtained for all participants.

\section{Material}

The same word stimuli as in Experiment 2 were used, but they were concatenated in lists alternating emotional and neutral stimuli. Four list types containing 20 lists each were created: (1) negative-frequent lists, composed of four negative words and two neutral words occurring at positions 2 and 4 [e.g., noyade (drowning) - orbite (orbit) - horreur (horror) - pile (battery) ennui (boredom) - avalanche (avalanche)], (2) positive-frequent lists, composed of four positive words and two neutral words occurring at positions 2 and 4 [e.g., triomphe (triumph) - plateau (tray) - lac (lake) - avenue (avenue) - encouragement (encouragement) - harmonie (harmony), (3) negative-infrequent lists, composed of four neutral words and two negative words occurring at positions 2 and 4 [e.g., mètre (meter) - assassin (murderer) - poivre (pepper) - faillite (bankruptcy) - camp (camp) - secrétaire (secretary)], and (4) positive-infrequent lists, composed of four neutral words and two positive words occurring at positions 2 and 4 [e.g., pièce (piece) sourire (smile) - tendance (tendency) - gaieté (cheerfulness) - estimation (estimation) - bocal (jar)]. Inter-item semantic relatedness was also formally assessed following the same procedure 
as above. Given the alternation of emotional and neutral stimuli, semantic relatedness should be near to 0 in all four lists. This was indeed observed, with mean modes of semantic relatedness amounting to $0.09 \pm 0.20$ for the first list type (four negative words) and $0.00 \pm 0.00$ for the three remaining list types.

Procedure

The lists were presented via headphones connected to a portable PC. The participants were instructed that they would hear 6 words presented via the headphones, and they were asked to recall the words in correct order immediately after presentation. After the participant's response, the examiner activated presentation of the next list. Each participant's responses were digitally recorded and stored on computer disk for later transcription and scoring. The eighty lists were presented in randomized order. For each condition, we determined the number of words correctly recalled, as well as item and order errors, as a function of emotional content.

Results and discussion

In order to determine the overall impact of mixed-list type on recall performance, we first compared recall performance for emotion-frequent and emotion-infrequent lists as a function of participant group and emotional condition (see Table 5 for descriptive statistics). We observed a main effect of mixed-list type, lists containing a minority of emotional words leading to highest recall performance, $\mathrm{F}(1,28)=8.49, \mathrm{MSE}=.01, \mathrm{p}<.01, \eta_{\mathrm{p}}^{2}=0.23$, as predicted by the preferential attentional grabbing hypothesis. We also observed a significant group effect, performance being overall higher for young participants, $F(1,28)=6.28, \mathrm{MSE}=.05, \mathrm{p}<.05, \eta_{\mathrm{p}}^{2}=0.18$. The effect of emotion type (positive vs. negative) was not significant, $F(1,28)=1.14, \mathrm{MSE}=.01, \mathrm{p}=.29, \eta^{2} \mathrm{p}$ 
$=0.04$. None of the interaction effects was significant. Next, we determined whether this enhanced recall performance for emotion-infrequent lists was due to the emotional words contained in these lists, by comparing recall performance for the emotional words in the emotion-infrequent lists to recall performance for the neutral words in emotion-frequent lists, thus comparing neutral and emotional words occurring in the same serial positions. We observed a highly significant effect of emotional content, $F(2,56)=12.57, \mathrm{MSE}=.01, \mathrm{p}<.001, \eta^{2} \mathrm{p}=0.31$, a significant effect of group, $\mathrm{F}(1,28)=6.45, \mathrm{MSE}=.05, \mathrm{p}<.05, \eta_{\mathrm{p}}^{2}=0.19$ as well as a significant group by emotional content interaction, $\mathrm{F}(2,56)=1.85, \mathrm{MSE}=.01, \mathrm{p}<.05, \eta_{\mathrm{p}}^{2}=0.12$ (see Figure 3a). Post-hoc comparisons (with Bonferroni corrections) revealed overall higher recall performance for both positive and negative words in emotion-infrequent lists relative to neutral words in emotion-frequent lists (all p's $<.05$ ); at the same time, while the difference between positive and neutral word recall was significant in both age groups, the difference between negative and neutral words was only significant in the group of young participants. Next, we determined whether there was also an advantage for recall of emotional words in emotionfrequent lists. In this analysis, we compared recall performance for the emotional words in the emotion-frequent lists to recall performance of the neutral words in emotion-infrequent lists, allowing us again to compare neutral and emotion words occurring in the same serial positions. This time, no significant effect of emotional content was observed, $F(2,56)=1.83$, MSE=.01, $\mathrm{p}=.17, \eta_{\mathrm{p}}^{2}=0.06$; only the effect of age group remained significant, $\mathrm{F}(1,28)=6.27, \mathrm{MSE}=.03$, $\mathrm{p}<.05, \eta_{\mathrm{p}}^{2}=0.18$ (see Figure $\left.3 \mathrm{~b}\right)$.

This latter result could be due either to the absence of an effect of emotional content on recall of emotional words in emotion-frequent lists or, as predicted by the weak attentional account, to an increase of recall performance for neutral words in the emotion-infrequent lists to 
which recall performance for the emotional words of emotion-frequent lists was compared. The current experiment is not suited to provide an unambiguous answer to this question since withinlist comparisons would be necessary, comparing recall for neutral and emotional words in the same list context. These are not possible since emotional and neutral words were not presented in the same serial positions within each list context. Although this issue will be explored explicitly in Experiment 4, we attempted to provide some answers to this question by comparing the results of the present experiment to the pure neutral and emotional lists presented in Experiment 2 on a between participant basis, by regrouping young and adult participant groups in order to increase the power of our analyses, by regrouping positive and negative emotion conditions, and by comparing recall performance for items stemming from the same serial positions in both experiments. However, none of these between-participant analyses was significant: emotional words in emotion-infrequent lists (Experiment 3) vs. neutral words (Experiment 2), $\mathrm{F}(1,58)<1$, $\mathrm{MSE}=.04, \mathrm{p}=.46, \eta_{\mathrm{p}}^{2}=0.01$; emotional words in emotion-frequent lists (Experiment 3 ) vs. neutral words (Experiment 2), $\mathrm{F}(1,58)<1, \mathrm{MSE}=.04, \mathrm{p}=.97, \eta_{\mathrm{p}}^{2}=0.01$. ; neutral words in emotion-frequent lists (Experiment 3) vs. neutral words (Experiment 2), F(1,58)<1, MSE=.01, $\mathrm{p}=.46, \eta_{\mathrm{p}}^{2}=0.01$; neutral words in emotion-infrequent lists (Experiment 3 ) vs. neutral words (Experiment 2), $\mathrm{F}(1,58)<1, \mathrm{MSE}=.01, \mathrm{p}=.90, \eta_{\mathrm{p}}^{2}=0.01$; neutral words in emotion-frequent lists (Experiment 3) vs. neutral words (Experiment 2), $F(1,58)<1, \mathrm{MSE}=.02, \mathrm{p}=.36, \eta_{\mathrm{p}}^{2}=0.01$; emotional words in emotion-frequent lists (Experiment 3) vs. emotional words (Experiment 2), $\mathrm{F}(1,58)<1, \mathrm{MSE}=.02, \mathrm{p}=.33 ., \eta_{\mathrm{p}}^{2}=0.02 ;$; emotional words in emotion-infrequent lists (Experiment 3) vs. emotional words (Experiment 2), $\mathrm{F}(1,58)<1, \mathrm{MSE}=.04, \mathrm{p}=.82, \eta_{\mathrm{p}}^{2}=0.01$.

\section{< INSERT TABLE 5 ABOUT HERE > $<$ INSERT FIGURE 3 ABOUT HERE >}


Next, we analyzed item and order errors as a function of emotional condition and list condition. In line with the preceding analysis, a first mixed ANOVA compared item errors for emotional words in emotion-infrequent lists relative to neutral words in emotion-frequent lists. A significant impact of emotional condition was observed, $F(2,56)=45.33, \mathrm{MSE}=.01, \mathrm{p}<.0001, \eta^{2} \mathrm{p}$ $=0.62$, item errors being less frequent for both positive and negative stimuli relative to neutral stimuli (Bonferroni post-hoc comparisons); a significant group effect was also observed, $\mathrm{F}(1,28)=6.87, \mathrm{MSE}=.05, \mathrm{p}<.05, \eta_{\mathrm{p}}^{2}=0.20$, item errors being more frequent in the elderly than then young adult group; the interaction term was not significant, $\mathrm{F}(2,56)=2.93$, MSE $=.01, \mathrm{p}=.06$, $\eta_{p}^{2}=0.09$ (see Table 6). On the other hand, when comparing item errors for emotional words in emotion-frequent lists relative to neutral words in emotion-infrequent lists, no effect of emotional condition was observed, $\mathrm{F}(2,56)<1, \mathrm{MSE}=.01, \mathrm{p}=.63, \eta_{\mathrm{p}}^{2}=0.02$; only a marginally significant group effect was observed, $\mathrm{F}(1,28)=3.81, \mathrm{MSE}=.03, \mathrm{p}=.06, \eta_{\mathrm{p}}^{2}=0.12$; the interaction term was not significant, $F(2,56)<1, \mathrm{MSE}=.01, \mathrm{p}=.46, \eta_{\mathrm{p}}^{2}=0.03$. It should be noted that the results reported in Table 6 may indicate that the effect we observed is mostly related to a change in error rates for neutral words between emotion-frequent and emotion-infrequent lists. However, a direct comparison between neutral words in the two list types or between emotional words in the two list types is difficult to interpret since the error rates stem from different serial position and a different number of serial positions. This will be addressed in Experiment 4. For order errors, we compared the proportion of order errors as a function of list condition. As discussed earlier, following the strong attentional account, exclusive attentional capture by emotional items in emotion-infrequent lists may lead to an item/order trade-off, disrupting encoding of serial order; on the other hand, following the weak attentional account, no negative impact on order encoding should be observed. We observed a main effect of emotion, $\mathrm{F}(1,28)=7.41$, MSE=.01, 
$\mathrm{p}<.05, \eta_{\mathrm{p}}^{2}=0.21$, lists containing negative words leading to a higher proportion of order errors, no effect of list type, $\mathrm{F}(1,28)=2.95, \mathrm{MSE}=.01, \mathrm{p}=.10, \eta_{\mathrm{p}}^{2}=0.10$, no effect of group, $\mathrm{F}(1,28)=3.76, \mathrm{MSE}=.01, \mathrm{p}=.06, \eta_{\mathrm{p}}^{2}=0.12$ but a significant three-way interaction, $\mathrm{F}(1,28)=5.00$, $\mathrm{MSE}=.01, \mathrm{p}<.05, \eta_{\mathrm{p}}^{2}=0.15$ (see Table 7). Post-hoc comparisons with Bonferroni corrections showed that these effects were driven mainly by a slightly higher proportion of order errors for young adults in lists containing negative words in emotion-infrequent relative to emotionfrequent lists.

\section{< INSERT TABLES 6 AND 7 ABOUT HERE >}

Overall, the results are in line with the predictions of preferential attentional capture in mixed list conditions: enhanced recall performance was observed for emotional words in an emotion-infrequent list context. Furthermore, the results appear to support the weak attentional account to a greater extent than the strong attentional account since recall performance was overall increased when recalling emotion-infrequent lists, as suggested by the main list type effect on recall performance and the low item error rates for neutral words in emotion-infrequent lists. Also, in line with this account, no reliable detrimental effect on order recall was observed. However, there remain a number of uncertainties. A recall advantage for neutral words in emotion-infrequent lists has yet to be directly shown by carrying out within-list comparisons, with emotional and neutral words occurring in the same serial positions. This will also allow for an unambiguous interpretation of the potential decrease of item errors for neutral words in emotion-infrequent lists. Furthermore, Experiments 1 and 2 have shown that linguistic knowledge underlying emotional words supports STM performance. Hence, we might nevertheless expect at least a mild effect of emotional content also for mixed lists containing a majority of emotional words relative to neutral words. No such effect could be demonstrated due 
to the design of the experiment, allowing no direct within-list comparisons of neutral and emotional words. All these issue were addressed in a final experiment using emotion-frequent and emotion-infrequent lists with emotional and neutral words occurring in all possible serial positions and allowing for direct, within-list comparisons.

\section{EXPERIMENT 4: ISR FOR MIXED LISTS OF EMOTIONAL WORDS IN YOUNG}

\section{PARTICIPANTS}

This final experiment explored recall performance for emotion-frequent and emotioninfrequent mixed lists, with emotional and neutral words occurring equally often in all serial positions for all list contexts, except for the first and final position (see Methods). Given that no consistent interactions with age group were observed in Experiments 2 and 3, only young participants were retained for this final experiment. Furthermore, we also included a control condition of pure lists containing exclusively neutral words. This control condition was administered to a second group of healthy young participants in order to avoid stimulus repetition effects for the neutral words occurring in both pure and mixed lists if administered to the same participant group. Finally, this experiment used visual rather than auditory presentation of stimuli.

\section{Participants}

40 young adults (20 female) aged between 18 and 30 years were recruited at the university campus. Mean age was 22 years. All participants were native French speakers and presented no history of developmental, learning or medical difficulties. Informed written consent was obtained for all participants. 
Material

Two-hundred forty positive or negative words and 240 neutral words were selected according to the same procedure as in the preceding experiment. In addition to the parameters already controlled in the preceding experiments (emotional valence, intensity, imageability, lexical frequency), we additionally controlled for number of letters given the visual presentation format used here, $\mathrm{F}(2,477)<1$, n.s (see Table 8 ). For the mixed lists, the words were pseudorandomly sampled without replacement and placed in any of the four following list conditions (while avoiding synonyms and direct semantic associates): (1) negative-frequent lists, composed of four negative words and two neutral words occurring either at positions 2 and 4 or at positions 3 and 5, (2) positive-frequent lists, composed of four positive words and two neutral words occurring either at positions 2 and 4 or at positions 3 and 5, (3) negative-infrequent lists, composed of four neutral words and two negative words occurring either at positions 2 and 4 or at positions 3 and 5, and (4) positive-infrequent lists, composed of four neutral words and two positive words occurring either at positions 2 and 4 or at positions 3 and 5. For each condition, there were 20 lists, with newly sampled lists for each participant, in order to randomize any residual semantic relatedness of items between lists and conditions. For the control condition of pure neutral word lists, 20 lists of neutral words were pseudo-randomly sampled from the pool of neutral words.

\section{< INSERT TABLE 8 ABOUT HERE >}

\section{Procedure}

The lists were presented on a computer screen, using E-Prime 1.0 software (Psychology Software Tools, Pittsburgh). The participants were instructed that they would see 6 words presented sequentially on the screen, and they were asked to recall the words in correct order immediately after presentation. The words were presented in white font on black background on 
the center of the screen. Presentation duration for each word was $1250 \mathrm{~ms}$, followed by a $250 \mathrm{~ms}$ duration black screen. After the sixth words, the instruction 'Recall' appeared on the screen and the participant started recalling the word lists. After the response, the participant pressed the space bar to activate presentation of the next trial. Each participant's responses were digitally recorded and stored on computer disk for later transcription and scoring. We determined the number of words correctly recalled as a function of list condition and stimulus type, as well as the amount of item errors and order errors.

Results and discussion

The impact of list type and stimulus type was explored using a repeated measures ANOVA, restricting the analyses to stimuli occurring in serial position from 2 to 5 , i.e. the positions where each stimulus type occurred equally often for each list context. We observed a main effect of list type, $F(1,39)=7.02, \mathrm{MSE}=.01, \mathrm{p}<.05, \eta_{\mathrm{p}}^{2}=0.15$, emotion-infrequent lists leading to overall higher performance levels relative to emotion-frequent lists, reproducing results of Experiment 3. However, we also observed a main effect of stimulus type: independently of list type, emotion words led to higher recall performance than neutral words, $\mathrm{F}(2,78)=7.97, \mathrm{MSE}=.01, \mathrm{p}<.001, \eta_{\mathrm{p}}^{2}=0.17$. The interaction term was not significant, $\mathrm{F}(2,78)<1$, $\mathrm{MSE}=.01, \mathrm{p}=.58, \eta_{\mathrm{p}}^{2}=0.01$. Post-hoc comparisons (with Bonferroni corrections for multiple comparisons) showed higher recall performance for positive words relative to neutral words, in both emotion-frequent and emotion-infrequent lists (see also Figure 4). As in Experiment 2 and 3, no reliable difference between recall performance for negative and neutral words was observed. The absence of an interaction between mixed list-type and stimulus type shows that not only emotional words, but also neutral words led to higher recall performance in emotion- 
infrequent mixed lists, as predicted by the weak attentional account. As in Experiment 3, we also performed between-participant comparisons, by comparing performance for emotion-infrequent lists in the experimental group to the control group which had been administered purely neutral word lists; as for the experimental group, we only considered performance for serial positions from 2 to 5 leading to a mean performance of $.66(\underline{ \pm} .13)$ for the neutral pure list control group. A between-group ANOVA revealed a significant difference between the neutral pure list control group and the experimental group when recalling positive words in emotion-infrequent lists, $\mathrm{F}(1,38)=5.13, \mathrm{MSE}=.02, \mathrm{p}<.05, \eta_{\mathrm{p}}^{2}=0.12$. As in the preceding Experiment 3 , all other betweengroup ANOVAs were non-significant: neutral pure list control group vs. the experimental group when recalling neutral words in emotion-infrequent lists, $\mathrm{F}(1,38)=1.97, \mathrm{MSE}=.02, \mathrm{p}=.17, \eta_{\mathrm{p}}^{2}$ $=0.05$; neutral pure list control group vs. the experimental group recalling positive words in emotion-frequent lists, $\mathrm{F}(1,38)=1.54, \mathrm{MSE}=.02, \mathrm{p}=.22, \eta_{\mathrm{p}}^{2}=0.04$; neutral pure list control group vs. the experimental group recalling neutral words in emotion-frequent lists, $F(1,38)<1$, $\mathrm{MSE}=.02, \mathrm{p}=.73, \eta_{\mathrm{p}}^{2}=0.01$.

\section{$<$ INSERT FIGURE 4 ABOUT HERE >}

In sum, as predicted by the weak attentional account, a reliable advantage for recalling positive emotional words was observed for an emotion-infrequent list context, as supported by both between-subject and within-subject analyses, together with an increase in recall accuracy for neutral words, as shown by within-subject analyses. At the same time, as predicted by the LTM support hypothesis, an advantage for recalling positive words was also observed in an emotion-frequent list context, as evidenced by significant emotion effects on recall performance irrespectively of list type. It must be noted that in both Experiments 3 and 4, between-participant analyses, comparing performance on pure and mixed lists, yielded few significant results despite 
sufficient power (sample size: $\mathrm{N}=30$ per group in Experiment 3), suggesting that the effects observed in mixed lists may be rather small. However, given the well documented and important inter-individual differences in STM performances, we cannot ascertain that the different groups were matched for basic STM capacity or general verbal abilities.

Finally, an analysis of item and order errors was performed. A repeated measures analysis assessed the impact of list type and stimulus type on item errors. We observed a main effect of list type, $\mathrm{F}(1,19)=16.14, \mathrm{MSE}=.01, \mathrm{p}<.001, \eta_{\mathrm{p}}^{2}=0.46$, item errors being less frequent in emotion-infrequent lists, and a main effect of stimulus type, $\mathrm{F}(2,38)=15.17$, MSE=.01, $\mathrm{p}<.0001$, $\eta_{p}^{2}=0.44$, positive words leading to a lower proportion of item errors that neutral or negative words, irrespectively of list-type; the interaction term was non-significant, $\mathrm{F}(2,38)=1.48$, $\mathrm{MSE}=.01, \mathrm{p}=.24, \eta_{\mathrm{p}}^{2}=0.07$ (see Table 9 for means and standard deviations). A last analysis explored the impact of list type and stimulus type on the proportion of order errors. No effect was significant: list type, $\mathrm{F}(1,19)<1, \mathrm{MSE}=.01, \mathrm{p}=.78, \eta_{\mathrm{p}}^{2}=0.01$, stimulus type, $\mathrm{F}(1,19)<1$, $\mathrm{MSE}=.01, \mathrm{p}=.96, \eta_{\mathrm{p}}^{2}=0.01$, interaction, $\mathrm{F}(1,19)=3.88, \mathrm{MSE}=.01, \mathrm{p}=.06, \eta_{\mathrm{p}}^{2}=0.17$ (Emotionfrequent negative: $.30 \pm .13$, Emotion-frequent positive lists: .26+.17, emotion-infrequent negative lists: $.27 \pm .18$, emotion-infrequent positive lists: .31+.21). In sum, as in Experiment 3, item errors, but not order errors were reliably influenced by list type. Furthermore, a reduction of item errors was observed for both neutral and emotional stimuli in emotion-infrequent lists. These results again support the weak but not the strong attentional account.

\section{<INSERT TABLE 9 ABOUT HERE >}

\section{GENERAL DISCUSSION}

Four experiments explored the impact of emotional valence on ISR performance, by comparing recall for neutral, positive and negative words. Experiments 1 and 2, using pure list 
designs, revealed a robust effect of emotional valence on item recall, as predicted by an increased LTM support for emotional words; this effect was most robust when comparing positive words to neutral words. Experiments 3 and 4, using a mixed list design, revealed increased recall performance for both emotional and neutral words in lists containing a minority of emotional words in line with a weak attentional account; at the same time, in line with the semantic LTM support hypothesis, an impact of emotional valence was also observed for lists containing a majority of emotional words, with enhanced recall accuracy for emotional words as compared to neutral words.

The emotional valence effect as new evidence for interactions between LTM and STM

The present study is the first to show a clear and robust effect of emotional content on STM word list recall performance. Only a few studies previously explored the impact of emotion on word list recall in a STM context, showing no effect of emotion on pure list recall (Kensinger \& Corkin, 2003), or showing an effect of positive valence on pure list recall but without controlling for semantic relatedness (Monnier \& Syssau, 2006), or showing an effect of emotion on mixed list recall (Ferré, 2002). Contrary to the studies by Kensinger and Corkin, which used only negative words, and by Monnier and Syssau, which used only positive words, the present study used both positive and negative words, and showed indeed the most reliable effect of emotional content on STM recall performance for positive words. Furthermore, none of these previous studies distinguished between semantic LTM and attentional factors in order to account for the impact of emotion on word list recall.

The pattern of effects we observed is highly consistent with the hypothesis of increased semantic LTM support for recall of emotional words: the advantage of recall for emotional words in pure and mixed lists was restricted to item recall, in line with the impact of other 
psycholinguistic effects on STM recall (word frequency, semantic relatedness; Nairne \& Kelley, 2004; Poirier \& Saint Aubin, 1995; Saint-Aubin \& Poirier, 1999). The question that arises here is the specific nature of increased semantic LTM support for emotional words. In the Introduction, we have shown that one specificity of emotional words is to have higher semantic relatedness since emotional words share a positivity-negativity dimension, as opposed to frequency and imageability matched neutral words (see also Talmi \& Moscovitch, 2004). However, in the present study we carefully controlled for semantic relatedness by organizing the items within lists in such a way that semantic relatedness was greatly reduced for both emotional and neutral lists, using a procedure similar to Tse and Altarriba (2009). Yet a robust effect of emotional valence was obtained, at least for positive words. It could be argued that this effect is due to the residual semantic relatedness, which, although very close to 0 , was nevertheless slightly higher for emotional lists relative to neutral lists. However, in the mixed lists (Experiments 3 and 4), this residual semantic relatedness was completely controlled, and yet a robust effect of emotional valence was still observed, especially when there were only a few emotional words. Hence, increased semantic relatedness is not sufficient to explain the increased LTM support for emotional words observed in this study. Another candidate, increased word imageability, can also be ruled out since this variable was carefully controlled for, as it was in previous studies (Tse \& Altarriba, 2009; Talmi \& Moscovitch, 2004; Monnier \& Syssau, 2008). Then how does the facilitated semantic LTM support for emotional words originate?

A first possibility, as already mentioned in the Introduction, is that emotional words form a specific semantic category that is richer (relative to neutral words) due to the additional activation of emotion-related semantic features, leading to more robust semantic activations in the language network. This interpretation is analogous to the dual-code hypothesis that has been proposed to explain the richer semantic processing for concrete, imageable words as opposed to 
abstract words (e.g., Walker \& Hulme, 1999; Paivio, 1986). A second possibility is that semantic representations for emotional words are more easily accessed or show decreased activation thresholds since these words refer to potentially life-enhancing or life-threatening dimensions: the rapid identification of the positive or negative features of a word could be important to avoid danger, to quickly identify sources of pleasure, and to quickly adapt behavior as a function of these emotional evaluations (e.g., Lang et al., 1997). A further possibility is that the specific semantic features of emotional words are directly connected to the motivational systems underlying emotion processing: the approach-appetitive system, for positive words, and the withdrawal-aversive system, for negative words (Bradley, 2000). Hence, when neutral words activate exclusively lexical and semantic language processing networks, emotion words in addition activate the motivational systems which, via their direct connections with the semantic network, strengthen the activation in the semantic network. This privileged connection between semantic and emotional processing is also suggested by a study by Huang et al. (2008), showing that preferential processing of emotional words presented at fast presentation rates is only achieved if the words have to be processed at a semantic level, rather than at a phonological level.

The second novel finding of the present study is that the impact of emotional content on STM is further modulated by attentional factors. Indeed, if increased LTM support was the only variable to explain higher STM recall for emotional words, then the effect of emotional valence should have been the weakest in the condition where only a minority of emotional words was presented. On the other hand, if the emotional effect was due to attentional variables only, the effect of emotional valence should have been the strongest in this same condition, and absent in the emotion-frequent and pure lists. In line with the attentional account, a robust effect of emotional valence was observed in mixed lists containing a minority of emotional words, 
relative to mixed lists containing a majority of emotional words (on a within-subjects basis in Experiments 3 and 4), and relative to pure neutral lists (on a between-subjects basis in Experiment 4). However, as already noted, only a weak version of the attentional account was supported by our data since not only recall of emotion words but also recall of adjacent neutral words was enhanced in emotion-infrequent lists. This pattern of results was predicted assuming that emotional words, by attracting attention, provide supplementary markers to the STM list, in addition to the standard start-of-list and end-of-list markers underlying enhanced recall for initial and final list positions (e.g., Henson, 1998). These additional markers facilitate the parsing of the STM list and hence lead to overall enhanced recall performance, as has been shown for other procedures loading into the focus of attention a minority of words via prosodic stressing procedures (e.g., Jones \& Yee, 1996; Reeves et al., 2000). A further assumption of this account is that attentional capture by emotion words is enhanced but not exclusive. In other words, emotional words receive preferential attentional focus, but without disturbing the processing of adjacent neutral words. Furthermore, it must be noted that this effect of weak attentional capture appears to work independently from the effect of LTM on emotion words in a STM task. Indeed, no strong evidence for additive effects of LTM and attentional capture was observed since recall of emotional words in emotion-infrequent lists, although higher relative to emotional words in emotion-frequent lists, was not higher relative to neutral words in the emotion-infrequent lists. Finally, the effect of emotion on mixed lists was not very strong, since an effect was observed reliably for within-participant comparison, but not for between-participant comparisons. Hence, overall, the present data provide evidence for a robust effect of LTM on recall of emotional words, as evidenced by enhanced recall performance for emotional words in pure lists and for emotion-frequent lists. In emotion-infrequent lists, the nature of the effect changes, with emotional words providing attentional markers facilitating encoding of the entire STM list. 


\section{Implications for models of STM}

At a theoretical level, the present data have a number of implications for current models of STM. On the one hand, as we have shown above, the emotional valence effect provides new evidence for theoretical models that consider strong interactions between verbal STM and linguistic LTM, especially at the level of semantic knowledge. The present data thus strengthen language-based models of STM such as those proposed by R. Martin et al. (1994), N. Martin \& Saffran (1992), Gupta (2003) and Majerus (2009). All these models consider that item information temporarily activates corresponding phonological, lexical and semantic representations: the richer and easier-to-access the underlying representations in the language network, the more robust their temporary activation, leading to an advantage of item recall in STM tasks. These models can account for the superiority of recall for emotion words in a pure list context, by adding the assumption that emotional-semantic features are represented in a specific part of the space of semantic representations (see also Figure 5). As we have discussed earlier, these representations are richer, show facilitated access relative to semantic representations for neutral words, and/or show additional connections with emotion processing systems; these additional connections will further enhance the stability of the activated semantic representations for emotional words. Hence, while greatly supporting existing language-based models of STM processing, the present study also shows that these models need some extension to take into account the specific semantic characteristics of emotional words and their connection with emotion-processing systems, as proposed in Figure 5. An alternative account of STM-LTM interactions considers that decaying STM traces are reconstructed, and this only at the moment of recall; this is the so-called redintegration process (Schweikert, 1993; Hulme et al., 1997; Walker \& Hulme, 1999). However, this position would also need to be extended to incorporate 
specific emotion-related semantic features that would be accessed at recall to reconstruct decaying traces for emotional words, in addition to more general semantic knowledge activated in common by emotional and neutral words.

\section{$<$ INSERT FIGURE 5 ABOUT HERE >}

A second theoretical implication of the present data is to highlight the additional influence of attentional factors when processing emotional words in a mixed list condition. Although many current STM models include interactions with LTM processes, only a few consider interactions with attentional factors. Cowan's embedded process framework is probably the most explicit STM model as regards the involvement of oriented attentional processes (e.g., Cowan, 1995). Like in the models presented in the previous paragraph, Cowan considers that STM capacity relies on the temporary activation of LTM representations, but in addition, Cowan assumes the existence of attentional processes (so-called focused attention) which maintain in the focus of attention the activated LTM representations. Following this model, STM capacity is driven by access to LTM representations and focused attention. With respect to the preferential attentional capture by emotion words, one would have to assume the existence of privileged connections between focused attention processes and semantic representations for emotion words, as proposed in Figure 5. When lists mixing emotional and neutral words are presented, emotional words, especially if there are only a few of them, will attract the attentional focus to a greater extent than neutral words (e.g., Cowan et al., 2001). These items, being in a heightened state of activation, will provide markers facilitating the encoding and retrieval of the items of the entire list. When there are many emotional words in the list, the attentional focus, which is of limited capacity (depending on the authors, the limit ranges between 1 and 4 items; Cowan, 1995; Oberauer \& Bialkova, 2009), will be attracted simultaneously by all these words, diluting the advantage of privileged attentional capture. 


\section{Conclusions}

Via the study of the impact of emotional valence on word list STM recall, the present study provides new evidence for the multi-determined nature of STM, showing that emotionalsemantic LTM is a further determinant factor of STM processing, in addition to the already well documented phonological and lexico-semantic LTM contributions to STM. In addition, the present study highlights the indirect contribution of attentional processes during the processing of emotional words, and during STM more generally. Overall, the present data call for integrative models of STM, accounting simultaneously for linguistic, emotional-linguistic and attentional variables as defining features of STM capacity. 


\section{ACKNOWLEDGMENTS}

Steve Majerus and Arnaud D’Argembeau are Research Associates funded by the Fonds de la Recherche Scientifique FNRS, Belgium. The authors thank Virginie Dupont, Alexandra Bockiau and Lucie Heiligenstein for their assistance in stimulus construction and data collection. This work was also supported by an IAP-Phase IV research grant No P6/29 from the Belgian Science Policy department, and a Concerted Research Action ARC 06/11-340 by the Ministry for Higher education and scientific research of the French-speaking Community, Belgium. 


\section{REFERENCES}

Algom, D., Chajut, E., \& Lev, S. (2004). A rational look at the emotional Stroop phenomenon: A generic slowdown, not a Stroop effect. Journal of Experimental Psychology: General, 133, 323-338.

Barnard, P. J., Ramponi, C., Battye, G., \& Mackintosh, B. (2005). Anxiety and the deployment of visual attention over time. Visual Cognition, 12,181-211.

Boersma, P. \& Weenink, D. (2001). Praat-A system for doing phonetics by computer. University of Amsterdam, The Netherlands: Institute of Phonetic Sciences.

Bradley, M. M. \& Lang, P. J. (1994). Measuring emotion: The self-assessment manikin and the semantic differential. Journal of Behavioral Therapy and Experimental Psychiatry, 25, 49-59.

Bradley, M. M. \& Lang, P. J. (1999). Affective norms for English words (ANEW). Gainesville, FL: University of Florida, NIMH Center for the Study of Emotion and Attention.

Bradley, M. M. (2000). Emotion and motivation. In L.G. Cacioppo, L. G. Tassinary, \& G. Berntson (Eds.), Handbook of Psychophysiology (pp. 602-642). New York: Cambridge University Press.

Burgess, N. \& Hitch, G. J. (1999). Memory for serial order: A network model of the phonological loop and its timing. Psychological Review, 106, 551-581. 
Christianson, S. A. \& Engelberg, E. (1999). Memory and emotional consistency: the MS Estonia ferry disaster. Memory, 7, 471-482.

Comblain, C., D’Argembeau, A., Van der Linden, M., \& Aldenhoff, L. (2004). The effect of ageing on the recollection of emotional and neutral pictures. Memory, 12, 673-684.

Cowan, N. (1995). Attention and memory: An integrated framework. New York: Oxford University Press.

Cowan, N. (2001). The magical number 4 in short-term memory: A reconsideration of mental storage capacity. Behavioral and Brain Sciences, 24, 87-185.

Damasio, A. R. (2001). Emotion and the human brain. In Unity of knowledge: The convergence of natural and human science, Annals of the New York academy of sciences (Vol. 935) (pp. 101-106). New York: New York Academy of Sciences.

DeLosh, E. L., \& McDaniel, M. A. (1996). The role of order information in free recall: Application to the word-frequency effect. Journal of Experimental Psychology: Learning, Memory, and Cognition, 22, 1136-1146.

Denburg, N., Buchanan, T.W., Tranel, D., \& Adolphs, R. (2003). Evidence for preserved emotional memory in normal older persons. Emotion, 3, 239-253.

Ferré, P. (2002). Advantage for emotional words in immediate and delayed memory tasks: Could it be explained in terms of processing capacity? The Spanish Journal of Psychology, 5, 78-89. 
Frankish, C. (1995). Intonation and auditory grouping in immediate serial recall. Applied Cognitive Psychology, 9, S5-S22.

Gathercole, S. E., Frankish, C. R., Pickering, S. J., \& Peaker, S. (1999). Phonotactic influences on short-term memory. Journal of Experimental Psychology: Human Learning and Memory, 25, 84-95.

Gaudreau, P. (2000). Vers une version française du PANAS : Analyses en composantes principales avant, pendant et après une compétition sportive. Actes du Congrès International de la Société Française de Psychologie du Sport, 61-62.

Gupta, P. (2003). Examining the relationship between word learning, nonword repetition and immediate serial recall in adults. Quarterly Journal of Experimental Psychology, 56A, 1213-1236.

Hadley, C.B., \& MacKay, D.G. (2006). Does Emotion Help or Hinder Immediate Memory? Arousal Versus Priority-Binding Mechanisms. Journal of Experimental Psychology: Learning, Memory and Cognition, 32, 79-88.

Henson, R. N. A. (1998). Short-term memory for serial order: The start-end model. Cognitive Psychology, 36, 73-137.

Huang, Y.-M., Baddeley, A. D., \& Young, A. E. (2008). Attentional capture by emotional stimuli is modulated by semantic processing. Journal of Experimental Psychology: Human Perception and Performance, 34, 328-339. 
Hulme, C., Maughan, S., \& Brown, G. D. (1991). Memory for familiar and unfamiliar words: Evidence for a long-term memory contribution to short-term memory span. Journal of Memory and Language, 30, 685-701.

Hulme, C., Roodenrys, S., Schweickert, R., Brown, G. D., Martin, S., \& Stuart, G. (1997). Word-frequency effects on short-term memory tasks: evidence for a redintegration process in immediate serial recall. Journal of Experimental Psychology: Learning, Memory, and Cognition, 23, 1217-1232.

Hulme, C., Stuart, G., Surprenant, A.M., Neath, I., Shostak, L., \& Brown, G. (2006). The distinctiveness of the word-length effect. Journal of Experimental Psychology: Learning, Memory, and Cognition, 32, 586-594.

Jones, M, \& Yee, W. (1993). Attending to auditory events: The role of temporal organization. In S. McAdams \& E. Bigand (Eds.), Thinking in sound: The cognitive psychology of human audition (pp. 69-112). New York: Oxford University Press.

Kanske, P. \& Kotz, S. A. (2007). Concreteness in emotional words: ERP evidence from a hemifield study. Brain Research, 1148, 138-148.

Kensinger, E.A., Brierley, B., Medford, N., Growdon, J.H., \& Corkin, S. (2002). Effects of normal aging and Alzheimer's disease on emotional memory. Emotion, 2, 118-134.

Kensinger, E. A. \& Corkin, S. (2003). Effect of negative emotional content on working memory and long-term memory. Emotion, 4, 378-393.

Kousta, S. T., Vinson, D. P., \& Vigliocco, G. (2009). Emotion words, regardless of polarity, have a processing advantage over neutral words. Cognition, 112, 473-481. 
Lang, P. J., Bradley, M. M., \& Cuthbert, B. N. (1990). Emotion, attention, and the startle reflex. Psychological Review, 97, 377-395.

Larser, R. J., Mercer, K. A., \& Balota, D. A. (2006). Lexical characteristics of words used in emotional Stroop experiments. Emotion, 6, 62-72.

MacKay, D., Shafto, M., Taylor, J. K., Marian, D. E., Abrams, L., \& Dyer, J. R. (2004). Relations between emotion, memory, and attention: Evidence from taboo Stroop, lexical decision, and immediate memory tasks. Memory and Cognition, 32, 474-488.

Majerus, S. \& Van der Linden, M. (2003). The development of long-term memory effects on verbal short-term memory: A replication study. British Journal of Developmental Psychology, 21, 303-310.

Majerus, S., Poncelet, M., Van der Linden, M., Albouy, G., Salmon, E., Sterpenich, V. et al. (2006). The left intraparietal sulcus and verbal short-term memory: Focus of attention or serial order? NeuroImage, 32, 880-891.

Majerus, S., Heiligenstein, L., Gautherot, N., Poncelet, M., \& Van der Linden, M. (2009). The impact of auditory selective attention on verbal short-term memory and vocabulary development. Journal of Experimental Child Psychology, 103, 66-86.

Majerus, S. (2009). Verbal short-term memory and temporary activation of language representations: the importance of distinguishing item and order information. In A.S.Thorn \& M. Page (Eds.), Interactions between short-term and long-term memory in the verbal domain (pp. 244-276). Hove, UK: Psychology Press. 
Martin, N. \& Saffran, E. M. (1992). A computational account of deep dysphasia: Evidence from a single case study. Brain and Language, 43, 240-274.

Martin, R. C., Shelton, J. R., \& Yaffee, L. S. (1994). Language processing and working memory: Neuropsychological evidence for separate phonological and semantic capacities. Journal of Memory and Language, 33, 83-111.

Mather, M. \& Carstensen, L. L. (2005). Aging and motivated cognition: The positivity effect in attention and memory. Trends in Cognitive Sciences, 9, 496-502.

Messina, D., Morais, J., \& Cantraine, F. (1989). Valeur affective de 904 mots de la langue française. Cahiers de Psychologie Cognitive/European Bulletin of Cognitive Psychology, 9, 165-187.

Monnier, C. \& Syssau, A. (2008). Semantic contribution to verbal short-term memory: Are pleasant words easier to remember than neutral words in serial recall and serial recognition? Memory and Cognition, 36, 35-42.

Mulligan, N.W. (2002). The generation effect: Dissociating enhanced item memory and disrupted order memory. Memory \& Cognition, 30, 850-861.

Nairne, J. S. \& Kelley, M. R. (2004). Separating item and order information through process dissociation. Journal of Memory and Language, 50, 113-133.

New, B., Pallier, C., Brysbaert, M., \& Ferrand, L. (2004). Lexique 2: A new French lexical database. Behavior Research Methods, Instruments, \& Computers, 36, 516-524. 
Oberauer, K., \& Bialkova, S., (2009). Accessing Information in Working Memory: Can the Focus of Attention Grasp Two Elements at the Same Time? Journal of Experimental Psychology: General, 138, 64-87.

Paivio, A. (1986). Mental representations: A dual coding approach. New York: Oxford University Press.

Paivio, A., Yuille, J. C., \& Madigan, S. A. (1968). Concreteness, imagery, and meaningfulness: Values for 925 nouns. Washington: American Psychological Association.

Pesta, B. J., Sanders, R. E., \& Murphy, M. D. (2001). Misguided multiplication: creating false memories with numbers rather than words. Memory and Cognition, 29, 478-483.

Poirier, M. \& Saint-Aubin, J. (1995). Memory for related and unrelated words: Further evidence on the influence of semantic factors in immediate serial recall. The Quarterly Journal of Experimental Psychology, 48A, 384-404.

Pratto, F. \& John, O. P. (1991). Automatic vigilance: The attention-grabbing power of negative social information. Journal of Personality and Social Psychology, 61, 380391.

Reeves, C., A. Schmauder, \& Morris, R.K. (2000). Stress grouping improves performance on an immediate serial list recall task. Journal of Experimental Psychology: Learning, Memory and Cognition, 26, 1638-1654.

Roodenrys, S., Hulme, C., Alban, J., \& Ellis, A. W. (1994). Effects of word frequency and age of acquisition on short-term memory span. Memory and Cognition, 22, 695-701. 
Saint-Aubin, J. \& Poirier, M. (1999). Semantic similarity and immediate serial recall: Is there a detrimental effect on order information? The Quarterly Journal of Experimental Psychology, 52A, 367-394.

Schweickert, R. (1993). A multinomial processing tree model for degradation and redintegration in immediate recall. Memory and Cognition, 21, 168-175.

Talmi, D. \& Moscovitch, M. (2004). Can semantic relatedness explain the enhanced memory for emotional words? Memory and Cognition, 32, 742-751.

Tse, C.S., \& Altarriba, J. (2009). The word concreteness effect occurs for positive, but not negative, emotion words in immediate serial recall. British Journal of Psychology, 100, 91-109.

Walker, I. \& Hulme, C. (1999). Concrete words are easier to recall than abstract words: Evidence for a semantic contribution to short-term serial recall. Journal of Experimental Psychology: Learning, Memory, and Cognition, 25, 1256-1271.

Watson, D., Clark, L.A., \& Tellegen, A. (1988). Development and validation of brief measures of positive and negative affects. The PANAS scales. Journal of Personality and Social Psychology, 54, 1063-1070. 
Table 1. Matching variables for neutral, positive and negative words in Experiment 1.

\begin{tabular}{llll}
\hline & Neutral & Positive & Negative \\
\hline Valence & $4.97(.23)$ & $2.30(.43)$ & $7.88(.39)$ \\
Intensity & $4.72(1.30)$ & $5.78(1.24)$ & $5.92(.40)$ \\
Lexical frequency & $41.00(43.80)$ & $35.74(33.02)$ & $44.48(71.10)$ \\
Word imageability & $5.70(1.83)$ & $5.60(1.52)$ & $5.43(1.36)$ \\
Number of syllables & $2.27(.72)$ & $2.21(.68)$ & $2.12(.75)$ \\
Duration (ms) & $894(203)$ & $867(154)$ & $902(269)$ \\
\hline
\end{tabular}


Table 2. Mean and standard deviations for recall accuracy in Experiment 1, as a function of list condition and serial position.

\begin{tabular}{|c|c|c|c|}
\hline & & Pure lists & \\
\hline & Neutral & Positive & Negative \\
\hline \multicolumn{4}{|c|}{ Serial position } \\
\hline 1 & $.99(.04)$ & $.98(.05)$ & $.96(.05)$ \\
\hline 2 & $.89(.12)$ & $.91(.10)$ & $.91(.12)$ \\
\hline 3 & $.85(.11)$ & $.84(.12)$ & $.83(.11)$ \\
\hline 4 & $.73(.15)$ & $.75(.14)$ & $.69(.20)$ \\
\hline 5 & $.61(.16)$ & $.68(.18)$ & $.74(.15)$ \\
\hline 6 & $.77(.15)$ & $.87(.14)$ & $.84(.15)$ \\
\hline Total & $.81(.18)$ & $.84(.21)$ & $.83(.18)$ \\
\hline
\end{tabular}


Table 3. Matching variables for neutral, positive and negative words in Experiment 2.

\begin{tabular}{lllll}
\hline & Neutral & Positive & Negative & F-value \\
\hline Valence (young participants) & $4.98(.16)$ & $2.24(.38)$ & $8.00(.29)$ & see text \\
Valence (elderly participants) & $4.6(.60)$ & $2.2(.50)$ & $8.2(.60)$ & see text \\
Intensity & $4.52(.44)$ & $5.13(1.58)$ & $6.32(1.09)$ & see text \\
Lexical frequency & $59.99(86.51)$ & $58.27(87.63)$ & $39.23(74.56)$ & see text \\
Word imageability & $5.70(1.85)$ & $5.50(1.59)$ & $5.40(1.39)$ & see text \\
Number of syllables & $2.04(.73)$ & $2.31(.84)$ & $2.30(.82)$ & see text \\
Duration (ms) & $790(190)$ & $801(190)$ & $820(181)$ & $\mathrm{F}(2,246)<1, \mathrm{n} . \mathrm{s}$. \\
Minimum pitch Syll 1(Hz) & $231(27)$ & $232(41)$ & $230(38)$ & $\mathrm{F}(2,246)<1, \mathrm{n} . \mathrm{s}$. \\
Maximum pitch Syll 1 (Hz) & $277(28)$ & $281(29)$ & $277(24)$ & $\mathrm{F}(2,246)<1, \mathrm{n} . \mathrm{s}$. \\
Minimum pitch Syll 2 (Hz) & $223(34)$ & $216(44)$ & $216(82)$ & $\mathrm{F}(2,140)=1.9, \mathrm{p}=.15$ \\
Maximum pitch Syll 2 (Hz) & $264(22)$ & $266(32)$ & $268(34)$ & $\mathrm{F}(2,140)<1, \mathrm{n} . \mathrm{s}$. \\
Minimum pitch Syll 3 (Hz) & $204(23)$ & $223(29)$ & $209(23)$ & $\mathrm{F}(2,16)=2.02, \mathrm{p}=.16$ \\
Maximum pitch Syll 3 (Hz) & $242(15)$ & $255(23)$ & $244(26)$ & $\mathrm{F}(2,16)=2.39, \mathrm{p}=.12$ \\
\hline
\end{tabular}


Table 4. Mean and standard deviations for recall accuracy in Experiment 2, as a function of list condition, serial position and age group.

\begin{tabular}{|c|c|c|c|}
\hline & & Pure lists & \\
\hline & Neutral & Positive & Negative \\
\hline \multicolumn{4}{|l|}{ Young adults } \\
\hline \multicolumn{4}{|c|}{ Serial position } \\
\hline 1 & $.95(.04)$ & $.97(.03)$ & $.94(.06)$ \\
\hline 2 & $.84(.10)$ & $.87(.08)$ & $.77(.13)$ \\
\hline 3 & $.79(.12)$ & $.73(.15)$ & $.67(.15)$ \\
\hline 4 & $.66(.18)$ & $.68(.23)$ & $.67(.24)$ \\
\hline 5 & $.58(.23)$ & $.75(.19)$ & $.65(.21)$ \\
\hline 6 & $.84(.10)$ & $.84(.10)$ & $.83(.11)$ \\
\hline Total & $.78(.21)$ & $.81(.24)$ & $.76(.21)$ \\
\hline \multicolumn{4}{|l|}{ Elderly adults } \\
\hline \multicolumn{4}{|c|}{ Serial position } \\
\hline 1 & $.83(.10)$ & $.84(.13)$ & $.78(.13)$ \\
\hline 2 & $.66(.15)$ & $.68(.14)$ & $.55(.15)$ \\
\hline 3 & $.42(.15)$ & $.54(.17)$ & $.45(.14)$ \\
\hline 4 & $.47(.19)$ & $.51(.21)$ & $.60(.20)$ \\
\hline 5 & $.51(.22)$ & $.66(.18)$ & $.59(.20)$ \\
\hline 6 & $.80(.10)$ & $.83(.11)$ & $.83(.12)$ \\
\hline Total & $.62(.21)$ & $.68(.24)$ & $.63(.23)$ \\
\hline
\end{tabular}


Table 5. Mean and standard deviations for recall accuracy in Experiment 3, as a function of list condition, serial position and age group.

\section{Mixed lists}

4 positive $\quad 4$ negative $\quad 2$ positive $\quad 2$ negative

Young adults

Serial position

\begin{tabular}{|c|c|c|c|c|}
\hline 1 & $.90(.12)$ & $.94(.13)$ & $.92(.13)$ & $.95(.11)$ \\
\hline 2 & $.77(.15)$ & $.77(.18)$ & $.85(.12)$ & $.83(.17)$ \\
\hline 3 & $.65(.15)$ & $.73(.15)$ & $.75(.17)$ & $.71(.14)$ \\
\hline 4 & $.60(.24)$ & $.54(.22)$ & $.63(.20)$ & $.66(.19)$ \\
\hline 5 & $.63(.20)$ & $.56(.18)$ & $.54(.21)$ & $.57(.21)$ \\
\hline 6 & $.86(.13)$ & $.79(.16)$ & $.84(.12)$ & $.83(.11)$ \\
\hline Total & $.73(.30)$ & $.72(.28)$ & $.75(.27)$ & $.76(.26)$ \\
\hline
\end{tabular}

Elderly adults

Serial position

\begin{tabular}{|c|c|c|c|c|}
\hline & $.83(.13)$ & $.83(.13)$ & $.82(.13)$ & $.89(.11)$ \\
\hline & $.66(.17)$ & $.64(.18)$ & $.77(.12)$ & $.63(.17)$ \\
\hline & $.50(.15)$ & $.55(.15)$ & $.59(.17)$ & $.63(.14)$ \\
\hline & $.48(.24)$ & $.45(.22)$ & $.52(.20)$ & $.52(.18)$ \\
\hline & $.59(.20)$ & $.53(.18)$ & $.43(.20)$ & $.43(.21)$ \\
\hline & $.82(.13)$ & $.77(.16)$ & $.76(.12)$ & $.79(.11)$ \\
\hline Total & $.65(.30)$ & $.63(.28)$ & $.65(.27)$ & $.65(.26)$ \\
\hline
\end{tabular}


Table 6. Mean and standard deviations for proportions of item errors in Experiment 3, regrouped as a function of stimulus and list condition.

\begin{tabular}{|c|c|c|c|c|c|c|}
\hline & \multicolumn{3}{|c|}{ Emotion-frequent lists } & \multicolumn{3}{|c|}{ Emotion-infrequent lists } \\
\hline & neutral & positive & negative & neutral & positive & negative \\
\hline Young adults & $.40(.14)$ & $.25(.07)$ & $.25(.08)$ & $.20(.12)$ & $.26(.12)$ & $.25(.12)$ \\
\hline Elderly adults & $.53(.17)$ & $.30(.12)$ & $.32(.12)$ & $.21(.15)$ & $.34(.14)$ & $.41(.16)$ \\
\hline
\end{tabular}


Table 7. Mean and standard deviations for proportions of order errors in Experiment 3, as a function of list condition.

\begin{tabular}{lllll}
\hline & & Mixed lists & & \\
& 4 positive & 4 negative & 2 positive & 2 negative \\
Young adults & $.20(.12)$ & $.18(.10)$ & $.19(.13)$ & $.27(.17)$ \\
Elderly adults & $.21(.15)$ & $.27(.21)$ & $.27(.20)$ & $.28(.18)$ \\
& & & & \\
\hline
\end{tabular}


Table 8. Matching variables for neutral, positive and negative words used in Experiment 4.

\begin{tabular}{llll}
\hline & Neutral & Positive & Negative \\
\hline Valence & $4.97(0.24)$ & $2.31(0.24)$ & $7.87(0.40)$ \\
Intensity & $4.51(0.48)$ & $5.08(1.62)$ & $6.17(1.18)$ \\
Lexical frequency & $35.27(35.98)$ & $37.74(42.78)$ & $36.65(62.70)$ \\
Word imageability & $5.86(1.89)$ & $5.46(1.60)$ & $5.51(1.89)$ \\
Number of letters & $6.99(1.66)$ & $7.22(1.89)$ & $6.96(1.73)$ \\
\hline
\end{tabular}


Table 9. Mean and standard deviations for proportions of item errors in Experiment 4, regrouped as a function of stimulus and list condition.

\begin{tabular}{lcll}
\hline & \multicolumn{3}{c}{ Stimulus type } \\
& neutral & positive & negative \\
\hline List type & & & \\
emotion-frequent & $.35(.12)$ & $.28(.09)$ & $.36(.09)$ \\
emotion-infrequent & $.26(.10)$ & $.23(.13)$ & $.30(.15)$ \\
& & & \\
\hline
\end{tabular}




\section{FIGURE LEGENDS.}

Figure 1. Item and order errors, as a function of list condition for Experiment 1.

Figure 2. Item and order errors, as a function of list condition and age group for Experiment 2. Figure 2A: young adults. Figure 2B: elderly adults.

Figure 3. Recall accuracy for emotional and neutral words in the mixed list conditions in Experiment 3. Figure 3A: emotion words in the emotion-infrequent lists versus neutral words in the emotion-frequent lists; Figure 3B: emotion words in the emotion-frequent lists versus neutral words in the emotion-infrequent lists.

Figure 4. Recall accuracy for emotional and neutral words in the mixed list conditions in Experiment 4.The dashed line indicates mean performance of the neutral pure list control group.

Figure 5. A proposal for a STM model integrating linguistic, emotional and attentional processing levels, as well as a serial order processing level. The dark-shaded area of overlap between the attentional focus and emotional-semantic representations indicates privileged attentional processing by emotion words. 
Figure 1.

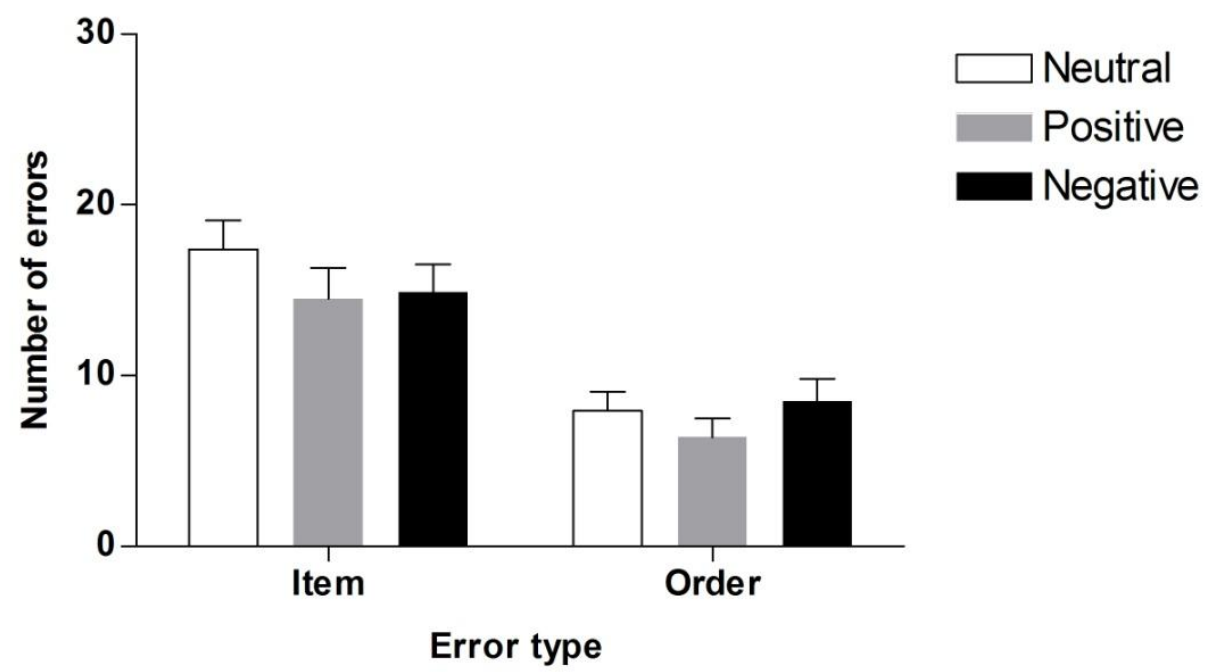


Figure 2.

Fig 2A

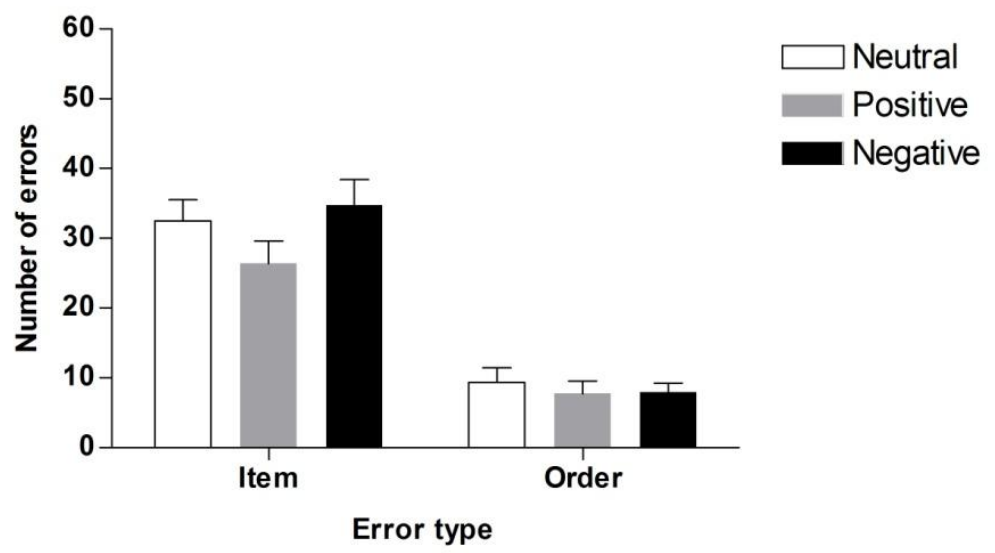

Fig 2B

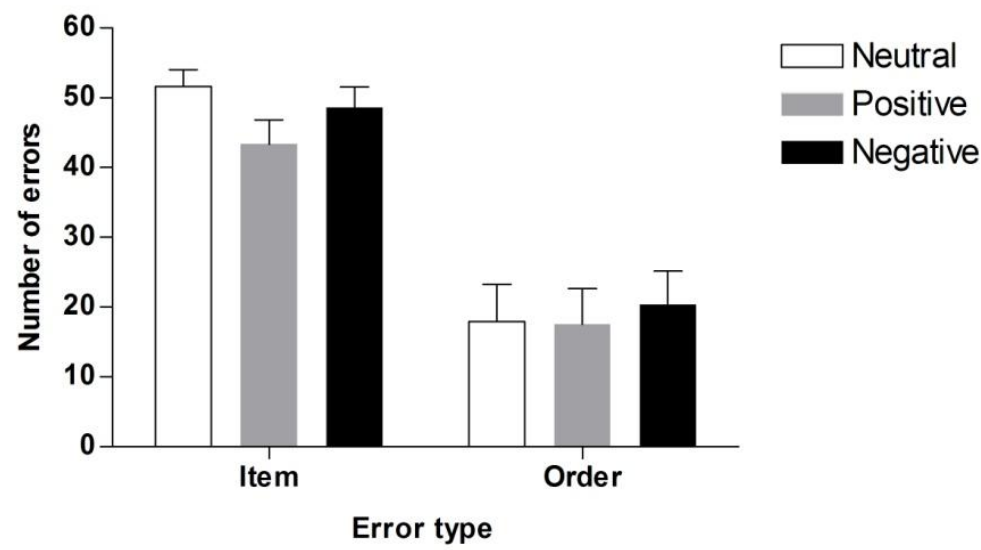


Figure 3

Fig $3 A$

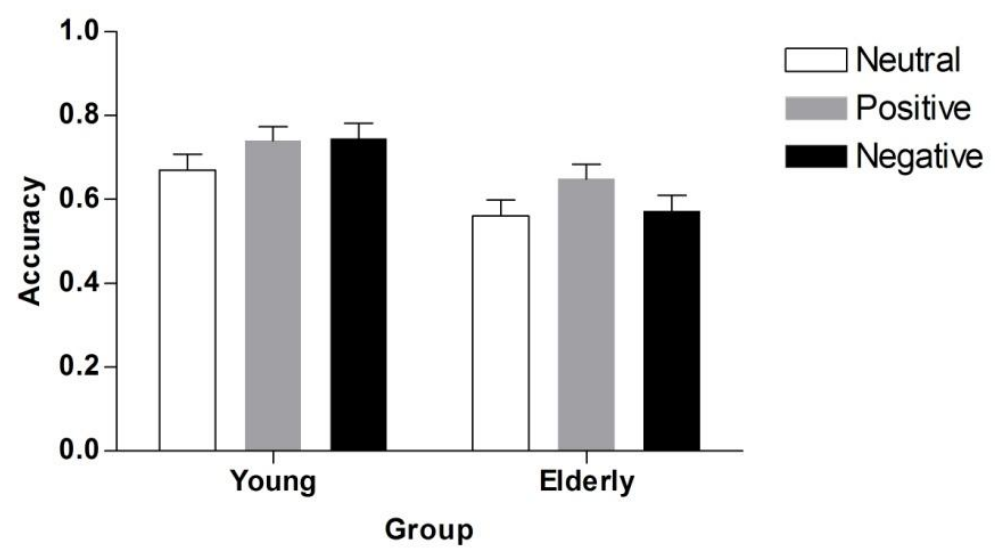

Fig 3B

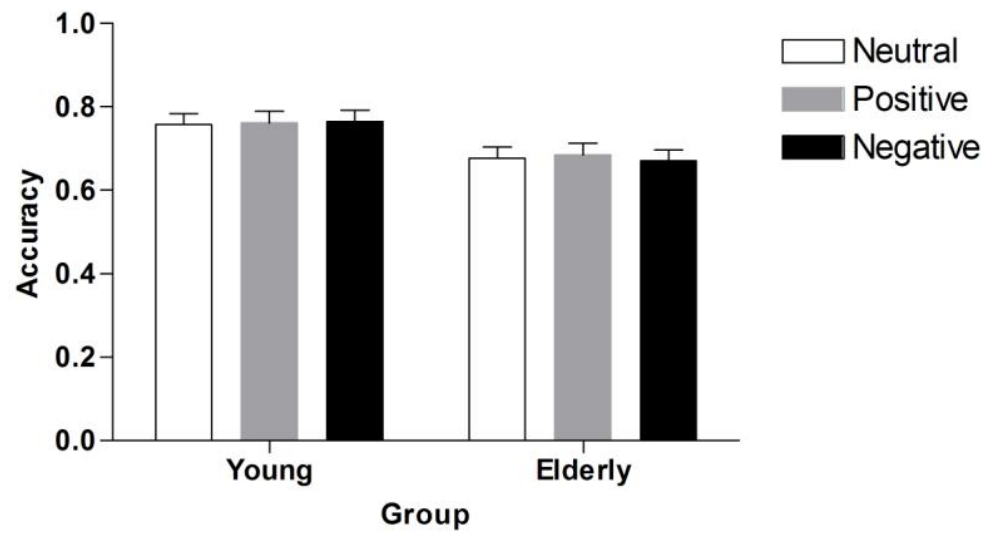


Figure 4.

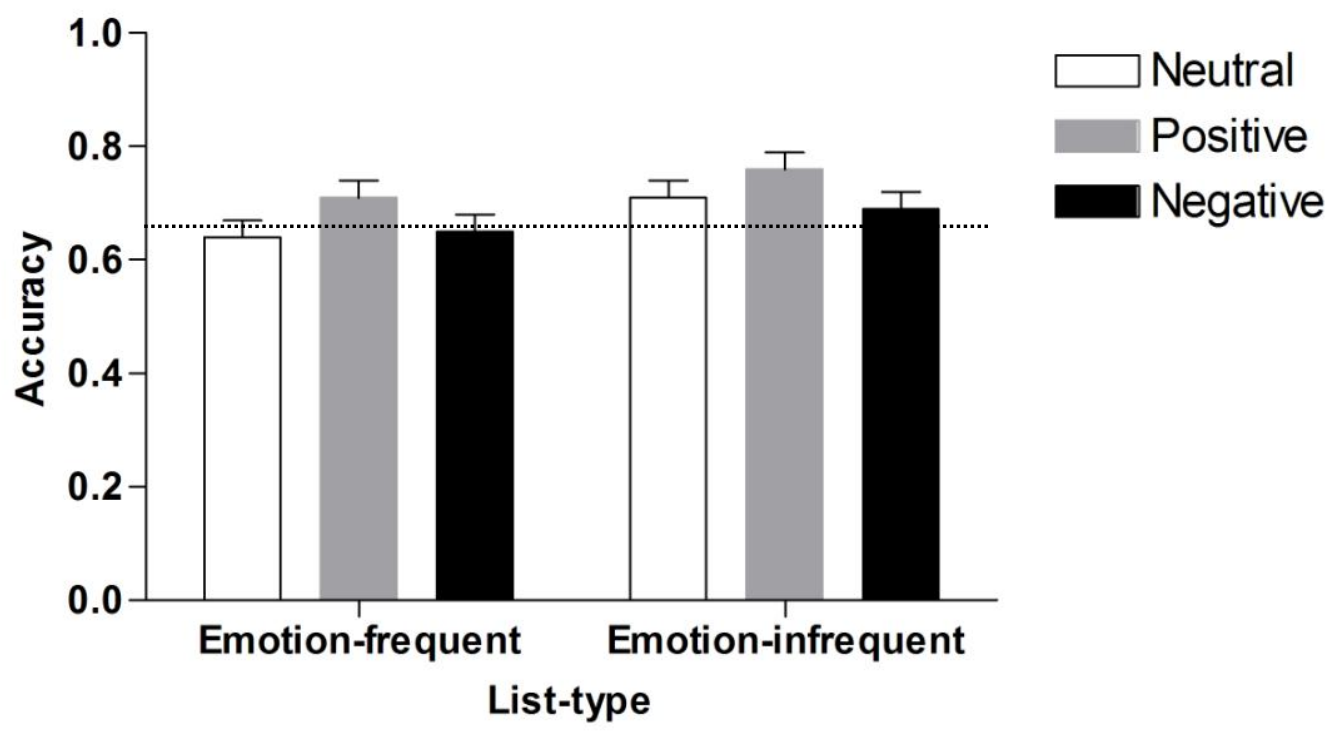


Figure 5.

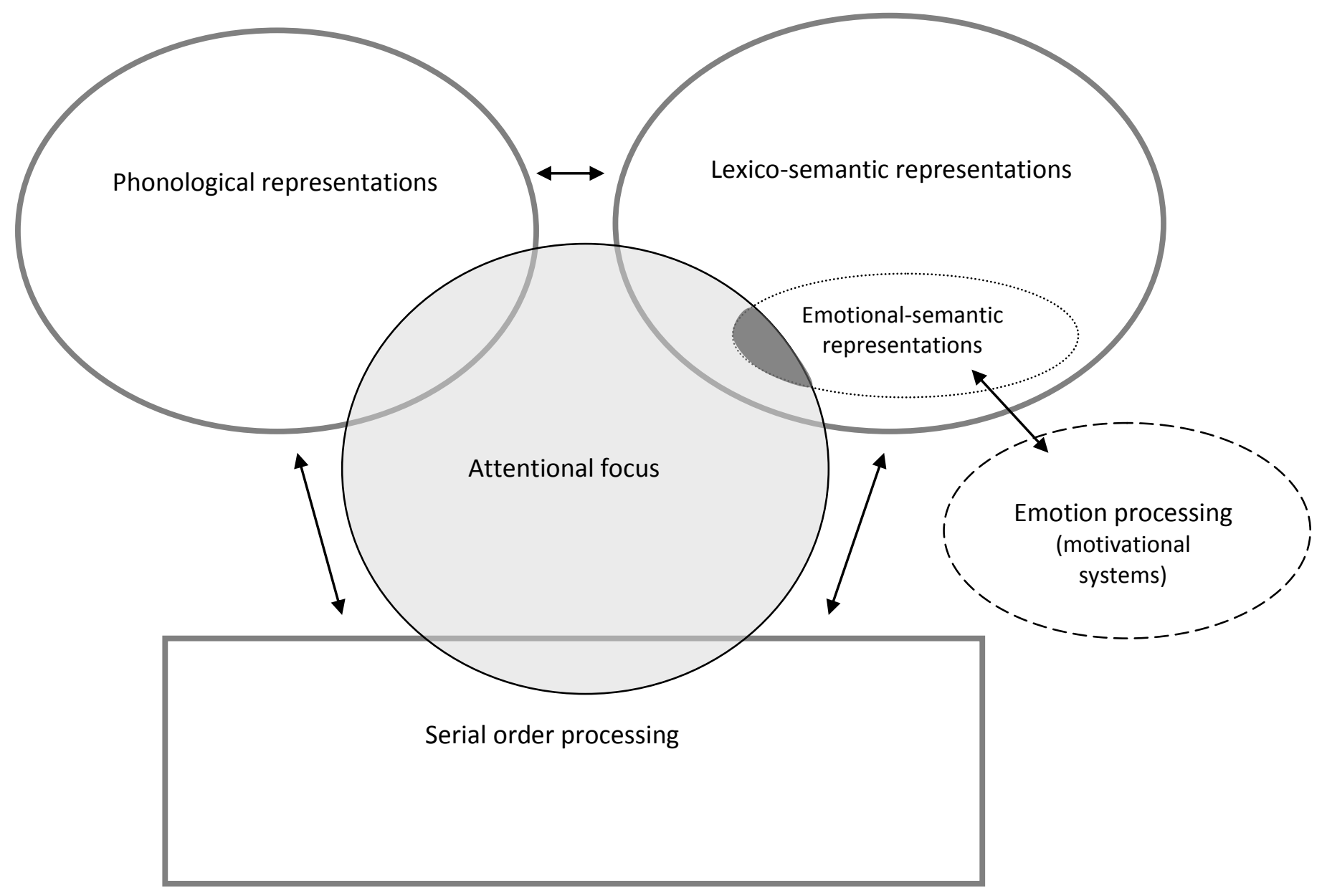

